OPEN ACCESS

Edited by:

David Pozo,

University of Seville, Spain

Reviewed by:

Girdhari Lal,

National Centre for Cell Science, India

Vladimir Mulens-Arias,

Pompeu Fabra University, Spain

*Correspondence:

Patrice N. Marche

patrice.marche@inserm.fr

Specialty section:

This article was submitted to

Molecular Innate Immunity,

a section of the journal

Frontiers in Immunology

Received: 08 June 2021 Accepted: 04 August 2021 Published: 23 August 2021

Citation:

Dey AK, Nougarède A, Clément $F$. Fournier C, Jouvin-Marche $E$, Escudé $M$, Jary D, Navarro FP and Marche PN (2021) Tuning the Immunostimulation Properties of Cationic Lipid Nanocarriers for Nucleic Acid Delivery.

Front. Immunol. 12:722411. doi: 10.3389/fimmu.2021.722411

\section{Tuning the Immunostimulation Properties of Cationic Lipid Nanocarriers for Nucleic Acid Delivery}

\author{
Arindam K. Dey ${ }^{1,2}$, Adrien Nougarède ${ }^{1,3}$, Flora Clément ${ }^{1,2,4}$, Carole Fournier ${ }^{1,2}$, \\ Evelyne Jouvin-Marche ${ }^{1,2}$, Marie Escudé ${ }^{1,3}$, Dorothée Jary ${ }^{1,3}$, Fabrice P. Navarro ${ }^{1,3}$ \\ and Patrice N. Marche ${ }^{1,2 *}$

\begin{abstract}
1 Univ. Grenoble Alpes, St Martin d'Hères, France, ${ }^{2}$ Institute for Advanced Biosciences, Research Center INSERM U1209, CNRS UMR5309, La Tronche, France, ${ }^{3}$ CEA, LETI, Division for Biology and Healthcare Technologies, Microfluidic Systems
\end{abstract} \\ and Bioengineering Lab, Grenoble, France, ${ }^{4}$ Univ. Grenoble Alpes, CEA, INSERM, IRIG, Biomics, Grenoble, France
}

Nonviral systems, such as lipid nanoparticles, have emerged as reliable methods to enable nucleic acid intracellular delivery. The use of cationic lipids in various formulations of lipid nanoparticles enables the formation of complexes with nucleic acid cargo and facilitates their uptake by target cells. However, due to their small size and highly charged nature, these nanocarrier systems can interact in vivo with antigen-presenting cells (APCs), such as dendritic cells (DCs) and macrophages. As this might prove to be a safety concern for developing therapies based on lipid nanocarriers, we sought to understand how they could affect the physiology of APCs. In the present study, we investigate the cellular and metabolic response of primary macrophages or DCs exposed to the neutral or cationic variant of the same lipid nanoparticle formulation. We demonstrate that macrophages are the cells affected most significantly and that the cationic nanocarrier has a substantial impact on their physiology, depending on the positive surface charge. Our study provides a first model explaining the impact of charged lipid materials on immune cells and demonstrates that the primary adverse effects observed can be prevented by fine-tuning the load of nucleic acid cargo. Finally, we bring rationale to calibrate the nucleic acid load of cationic lipid nanocarriers depending on whether immunostimulation is desirable with the intended therapeutic application, for instance, gene delivery or messenger RNA vaccines.

\section{Keywords: nanostructured lipid carrier, antigen presenting cells, nucleic acid delivery, immunotoxicity assessment, surface charge (zeta potential)}

\section{INTRODUCTION}

In recent years, advances in field of nanotechnology have demonstrated potential for precision medicine. For instance, lipid nanoparticles (LNPs) can be used for the targeted delivery of therapeutic molecules, increasing their bioavailability and pharmacokinetic properties beyond the Lipinski rules (1). Indeed, the development of nucleic acid therapeutics has long been hampered by 
the inherent hydrophilic nature, large size, and poor membrane permeability of nucleic acids (2). LNPs can be a potent alternative to viral-mediated nucleic acid delivery, with an extensive range of applications such as RNA interference (RNAi) therapy or RNA-based vaccines through intracellular delivery, respectively, of short interfering RNA (siRNA) or messenger RNA (mRNA) (3).

One of the primary advantages associated with LNPs is their biocompatibility that enables their use in vivo for human therapy $(4,5)$. LNPs are made of two major components: a lipid phase and a water phase containing surfactants. LNPs are generally divided into liposomes with an aqueous core or other LNPs; the latter could be solid lipid nanoparticles (SLNs) with a solid core and nanostructured lipid carriers (NLCs) featuring a core that is a mixture of solid and molten lipids (6). This subclass of LNPs was initially designed to improve the colloidal stability of lipid carriers and increase the drug payload into the core by controlling the release profile (7). Moreover, they are considered advantageous because their manufacturing processes can be easily scaled up for large production (8).

Due to the nature of their lipid core, these particles are not well adapted for nucleic acid encapsulation. The loading of biomacromolecules such as siRNA or mRNA, therefore, occurs through the association with their shell either by chemical modifications of Polyethylene glycol (PEG) residues (9) or by incorporation of cationic lipids at the level of phospholipid monolayer, thus allowing electrostatic interactions with negatively charged nucleic acids (9-12). The most chosen cationic lipids are quaternised cationic lipids, such as Dioleoyl3-trimethylammonium propane (DOTAP), which are added to the formulation at the appropriate ratio (13). The NLCs with DOTAP present thereby a globally positive charge; thus, their toxicity and their impact on the immune systems need to be assessed. A previous study has reported that positively charged nanocarriers induce some systemic toxicity and proinflammatory effects (14). The microenvironment is known to drive distinct antigen-presenting cell (APC) fates by affecting functions of macrophages and dendritic cells (DCs) by activating different metabolic pathways. For example, while lipopolysaccharides (LPS) classically activated macrophages (M1), displaying pro-inflammatory activity, rely on glycolysis, Interleukin 4 (IL-4) alternatively activated macrophages (M2),

\footnotetext{
Abbreviations: APCs, antigen presenting cells; DCs, dendritic cells; LNPs, Lipid nano particles; RNAi, RNA interference; siRNA, small interfering RNA, mRNA, messenger RNA; SLN, solid lipid nanoparticles; NLCs, nanostructured lipid carriers; PEG, polyethylene glycol; DOTAP, Dioleoyl-3-trimethylammonium propane; M1, pro-inflammatory macrophages; M2, anti-inflammatory macrophages; BMDCs, bone marrow derived dendritic cells; BMDMs, bone marrow derived macrophages; cNLCs, cationic lipid carrier; CD, cluster of differentiation; FAO, fatty acid oxidation; ECAR, extracellular acidification rate; IL, interleukin; PDI, polydispersity index; DLS, dynamic light scattering; ELS, electrophoretic light scattering; SD, standard deviation; CBA, cytometric Bead Array; LDH, lactate dehydrogenase; LPS, lipopolysaccharide; LC, lipid nanocarrier; OCR, oxygen consumption rate; OXPHOS, oxidative phosphorylation; NLC, nano structured lipid carrier; cNLCs, cationic nanostructured lipid carriers; nNLCs, neutral nanostructured lipid carriers; NPs, nano particles; ROS, reactive oxygen species; SLN, solid lipid nanoparticles; TNF, tumor necrosis factor.
}

displaying anti-inflammatory activity, primarily utilise fatty acid oxidation (FAO) and oxidative phosphorylation (OXPHOS) (15). DCs, like macrophages, respond differently in the presence of LPS and IL4 (16).

The exposition to cationic lipid carriers (cNLCs) has been shown to affect the functions of APCs. For instance, cNLCs were shown to activate bone-marrow-derived dendritic cells (BMDCs) partially by inducing the expression of two costimulatory molecules, CD80 and CD86, but without inducing the secretion of pro-inflammatory cytokines (17).

DOTAP itself could interact directly with ligands on the surface of the immune system (18). In the cationic NLCs formulation, we describe here that the phospholipid layer incorporating cationic lipids is covered by a dense PEGylated coating that contributes to the stability and also is known to reduce the interaction with proteins and other biological entities $(14,19,20)$.

Moreover, how the positive charge of lipid particles modulates the metabolic fitness of APCs and how this is related to the cellular function have not yet been elucidated. Therefore, understanding the impact of positively charged particles on immune responses and particularly on APCs metabolism, fate and cytokine secretion is crucial to control the use of nanocarriers fully.

In the present study, we analysed the effect of NLCs surface charge on primary APCs using BMDCs and bone-marrowderived macrophages (BMDMs), as cellular models. We evaluated the impact of neutral lipid carriers (nNLCs) and cNLCs on the secretion of different signalling factors and mitochondrial metabolism and glycolysis. Furthermore, we used negatively charged siRNA to reverse the net charge on cNLCs and evaluate the effect of different surface charges on cell function.

\section{MATERIALS AND METHODS}

\section{Cell Culture}

The murine macrophage cell line (J774.1A) was purchased from ATCC; the cells were cultured in Dulbecco's modified Eagle's medium (DMEM) supplemented with 10\% fetal bovine serum and $1 \%$ penicillin-streptomycin.

As previously described (21), BMDCs were generated from the bone marrow extracted from C57BL/6 mice (Charles River, l'Arbresle, France). Bone marrow cells were isolated by flushing from the tibia and femur. Erythrocytes and GR1 positives cells were removed by incubating with Ly-6G/Ly-6C (BD Pharmingen, \#553125) and TER-119 (BD Pharmingen, \#553672) antibodies, and the remaining negatively sorted cells were isolated using Dynabeads isolation kit (ThermoFisher, \#11047) by magnetic cell sorting; then the remaining negatively sorted cells were resuspended at $5 \times 10^{5}$ cells $/ \mathrm{ml}$ in complete Iscove's modified Dulbecco's medium supplemented with Granulocyte-macrophage colony-stimulating factor (GM-CSF) (PeproTech, \#315-03), FLT-3L (PeproTech, \#250-31L) and Interleukin 6 (IL-6) (Peprotech, \#216-16) according to Table 1. The transformation of the progenitors into fully active DCs was performed over a 10-day time frame. 
TABLE 1 | Concentration of GM-CSF, FLT-3L and IL-6 for BMDCs culture.

\begin{tabular}{|c|c|c|c|c|c|c|}
\hline \multicolumn{5}{|c|}{ Cells are cultured in a 100-mm TC-treated cell culture dish with $15 \mathrm{~mL}$ culture media } & Day 7 & Day 10 \\
\hline Cell concentration & & $0.6 \times 10^{6} / \mathrm{mL}$ & $0.5 \times 10^{6} / \mathrm{mL}$ & $0.5 \times 10^{6} / \mathrm{mL}$ & $0.5 \times 10^{6} / \mathrm{mL}$ & According to cell plating \\
\hline Supplement & $\mathrm{IL}-6$ & $5 \mathrm{ng} / \mathrm{mL}$ & $2.5 \mathrm{ng} / \mathrm{mL}$ & $2.5 \mathrm{ng} / \mathrm{mL}$ & - & - \\
\hline & FLT-3L & $50 \mathrm{ng} / \mathrm{mL}$ & $40 \mathrm{ng} / \mathrm{mL}$ & $30 \mathrm{ng} / \mathrm{mL}$ & $25 \mathrm{ng} / \mathrm{mL}$ & $25 \mathrm{ng} / \mathrm{mL}$ \\
\hline & GM-CSF & $5 \mathrm{ng} / \mathrm{mL}$ & $5 \mathrm{ng} / \mathrm{mL}$ & $5 \mathrm{ng} / \mathrm{mL}$ & $5 \mathrm{ng} / \mathrm{mL}$ & $5 \mathrm{ng} / \mathrm{mL}$ \\
\hline
\end{tabular}

Culture of BMDCs: BMDCs were seeded into a 100-mm TC-treated cell culture dish with $15 \mathrm{~mL}$ culture media. Culture media is supplemented with variable concentrations of GM-CSF, FLT-3L and IL-6 on day 0, day 3, day 5, day 7 and day 10 to harvest fully differentiated BMDCs on day 11.

BMDMs were also generated from bone marrow extracted from C57BL/6 mice as previously described (22). Briefly, the erythrocytes were removed by the RBC lysis buffer, and the remaining cells were cultured in a complete DMEM with $20 \%$ L929 (Sigma, \#85011425) in conditioned medium (source of macrophage colony-stimulating factor) for 7 days.

\section{Cationic and Neutral Lipid Nanocarriers}

nNLCs and cNLCs were prepared as described in the previous study (23). Briefly, for nNLCs, a lipid phase was prepared containing triglycerides (Suppocire NB, Gattefossé and superrefined soybean oil, Croda Uniqema) and phospholipids (Lipoid SPC3, Lipoid). For cNLCs, the same lipid phase supplemented with the cationic lipid DOTAP (1,2-dioleoyl-3trimethylammonium-propane chloride, Avanti Polar Lipids) and fusogenic lipid DOPE (1,2-dioleoyl-sn-glycero-3phosphoethanolamine, Avanti Polar Lipids) were used. When indicated, Dil lipophilic dye (D282, ThermoFisher) was added to the lipid phase to enable fluorescence detection of nNLCs. A second aqueous phase containing the PEGylated surfactant PEG40 Stearate (Myrj S40, Croda Uniqema) was prepared in Phosphate-buffered saline (PBS) (\#806552, Sigma). Both lipid and aqueous phases were mixed together through highfrequency sonication. Lipid nanoparticles are purified by dialysing in 100 volumes of LNP buffer: $154 \mathrm{mM} \mathrm{NaCl}, 10$ mM HEPES, and $\mathrm{pH} 7.4$ using endotoxin-free ultra-pure water (TMS-011-A, Sigma) and 12-14 kDa MW cut-off membranes (ZelluTrans/Roth T3). Finally, the LNP solution was sterilised by filtrating through a $0.22-\mu \mathrm{m}$ millipore membrane. A putative structure depicting each component of cNLCs and nNLCs is presented in Supplementary Figure 1.

\section{Nanoparticle Uptake Assay}

For nanoparticle uptake assays, $0.5 \times 10^{5}$ cells $/ \mathrm{mL}$ of BMDCs and BMDMs were seeded into a 4-well Lab-Tek chambered coverslip. After $24 \mathrm{~h}$ of growth, the cells were incubated with both Dillabelled nanocarriers, cNLCs and nNLCs, for $24 \mathrm{~h}$ at $37^{\circ} \mathrm{C}$ with $5 \%$ $\mathrm{CO}_{2}$. Nanocarrier accumulation inside cells was monitored by time-lapse microscopy using a spinning disk confocal microscope (Andromeda, TILL-FEI). The Dil-labelled nanocarriers were visualised using the lipophilic dye excitation wavelength of 514 $\mathrm{nm}$ while plasma membranes were labelled with FITC-conjugated cholera toxin (Sigma, C1655) and visualised at the excitation wavelength of $488 \mathrm{~nm}$. After acquisition, the images were processed in Icy 2.0.3.0 software, and spectral deconvolution was performed using NIS 5.20 .01 software.

\section{Physical Characterisation of NLCs}

The hydrodynamic diameter and polydispersity index (PDI) of the NLCs were determined by dynamic light scattering (DLS), and the zeta potential was determined by electrophoretic light scattering (ELS) using a Zetasizer Nano ZS instrument (Malvern). The hydrodynamic diameter and PDI were measured with a dispersion of $1 \mathrm{mg} / \mathrm{mL}$ NLCs in PBS while the zeta potential was measured with a dispersion of $1 \mathrm{mg} / \mathrm{mL}$ NLCs in $1 \mathrm{mM} \mathrm{NaCl}$. Each assay was performed in three replications at $25^{\circ} \mathrm{C}$. A table showing the size, PDI and zeta potential of both NLCs is documented in Supplementary Figure 1.

\section{Complexation of cNLCs With Nucleic Acid}

In the complexation of cNLCs with model nucleic acid, all-star negative control siRNA (siMock) was carried out in PBS. The required volume for siMock was calculated according to the desired N/P ratios (ratio of positively-chargeable polymer amine $(\mathrm{N}=$ nitrogen) groups to negatively-charged nucleic acid phosphate (P) groups) at a constant concentration of the cNLCs nanocarrier $(100 \mu \mathrm{g} / \mathrm{mL})$. The cNLCs carrier and diluted siMock were gently homogenised by pipetting and kept for $10 \mathrm{~min}$ at room temperature before immediate use for downstream experiments.

\section{Incubation With Nanoparticles}

For cell culture, 12, 24 and 96 cell culture microplates manufactured by Falcon ${ }^{\circledR}$ or seahorse XFe96 were used. Cells were seeded at a concentration of $10^{6}$ cells $/ \mathrm{mL}$ and cultured for $24 \mathrm{~h}$. They were incubated for $24 \mathrm{~h}$ with nNLCs or cNLCs at a concentration ranging from 20 to $100 \mu \mathrm{g} / \mathrm{mL}$. Cells were subsequently washed and stimulated with LPS $(2 \mu \mathrm{g} / \mathrm{mL})$ or IL-4 $(20 \mathrm{ng} / \mathrm{mL})$ for another $24 \mathrm{~h}$. Finally, the impact of the two nanocarriers on BMDMs and BMDCs was assayed using various parameters, such as viability, phagocytosis, activation, cytokine secretion, nitric oxide $(\mathrm{NO})$ production, reactive oxygen species (ROS) production and glycolysis or mitochondrial metabolism.

\section{Toxicity Assessment}

Toxicity was measured by quantifying the cell viability using the CytoTox-ONE ${ }^{\mathrm{TM}}$ Homogeneous Membrane Integrity Assay kit (Promega, G7891) according to the manufacturer's protocol. Briefly, the lysis solution $(2 \mu \mathrm{l}$ of lysis solution per $100 \mu \mathrm{l}$ original volume) was used as a positive control for lactate dehydrogenase $(\mathrm{LDH})$ release. A volume of $100 \mu \mathrm{L}$ of CytoTox-ONE ${ }^{\mathrm{TM}}$ reagent was added to each well, before homogenisation on a shaker for 30 seconds and followed by 
incubation for another $10 \mathrm{~min}$ in the dark. After that, stop solution $(50 \mu \mathrm{L})$ was added to each well, and the plate was placed on the shaker for another 10 seconds. Finally, their fluorescence was recorded at an excitation wavelength of $560 \mathrm{~nm}$ and an emission wavelength of $590 \mathrm{~nm}$ using a CLARIOstar ${ }^{\circledR}$ microplate reader (BMG LABTECH).

\section{Phagocytosis Assay}

Nanocarrier-exposed macrophages (BMDMs and J774.1A cells) and BMDCs were incubated at a ratio of 10 microspheres per cell for $6 \mathrm{~h}$ with $1.0-\mu \mathrm{m}$ FluoSpheres ${ }^{\circledR}$ carboxylate-modified microspheres (ThermoFisher, F8851) labelled with a red fluorescent dye (580 nm excitation and $605 \mathrm{~nm}$ emission). Cells were analysed by flow cytometry with an Accuri C6 instrument (Becton-Dickinson), and the analysis was performed by the FCS Express V5 software (De Novo Software).

\section{Cell Activation}

Nanocarrier-exposed BMDCs and BMDMs were stimulated for $24 \mathrm{~h}$ using $2 \mu \mathrm{g} / \mathrm{mL}$ LPS from Escherichia coli. Supernatants were collected for downstream cytokine immunoassay. After blocking the Fc receptor (BD Pharmingen, 553142) to reduce nonspecific binding, BMDCs and BMDMs were stained for $\mathrm{APC} / \mathrm{Cy} 7$ conjugated CD11b (Ozyme, BLE101226) and PE/Cy7 conjugated CD11c (Ozyme, BLE117318) or PE/Cy7 conjugated CD11b (Ozyme, BLE101216) and APC/Fire ${ }^{\text {TM }} 750$ conjugated F4/80 (Ozyme, BLE123152), respectively. To evaluate the cell activation, BMDCs and BMDMs were stained with Alexa Fluor ${ }^{\circledR}$ 488 conjugated anti-IAb (Ozyme, BLE116410) and PE conjugated CD86 (Ozyme, BLE105008) antibodies. In both cases, live cells were selected by negative 7-aminoactinomycin D (7AAD; BD Pharmingen, 559925) staining and analysed by flow cytometry using an LSR II instrument (Becton-Dickinson). The proportion of activated cells was quantified using FCS Express V5 software.

\section{Cytokine Immunoassays}

Cytokine production was measured from cell culture supernatants with cytometric bead array (CBA; BD Pharmingen, 552364) using a mouse inflammation kit against IL-6, IL-12p70, MCP-1, TNF $\alpha$, IL-10 and IFN $\gamma$. Results were acquired by flow cytometry using a BD LSR II instrument and analysed with FCAP Array Software v3.0 (BD Pharmingen, 652099).

\section{NO and ROS Production}

NO produced by BMDMs and BMDCs was determined by measuring nitrite concentration in cell culture media by Griess assay. Briefly, $50 \mu \mathrm{L}$ of cell supernatant was transferred to a 96well plate and incubated with an equal volume of sulphanilamide (Sigma, S9251) and N-alpha-naphthyl-ethylenediamine (Sigma, 222488) solutions, respectively, for $10 \mathrm{~min}$ each, protected from light. Optical density was measured at $540 \mathrm{~nm}$ using a CLARIOstar ${ }^{\circledR}$ microplate reader, and sample nitrite concentration was determined using a standard curve. ROS production by BMDMs and BMDCs was determined by ROSGlo ${ }^{\mathrm{TM}} \mathrm{H}_{2} \mathrm{O}_{2}$ assay kit (Promega, G8821). The cells were cultured at $5 \times 10^{4} \mathrm{cell} / \mathrm{mL}$ concentration in a 96-well plate, exposed to nanocarriers for $24 \mathrm{~h}$ and stimulated with $2 \mu \mathrm{g} / \mathrm{mL}$ of LPS. A volume of $20 \mu \mathrm{L}$ of $\mathrm{H}_{2} \mathrm{O}_{2}$ substrate solution was added to each well before $6 \mathrm{~h}$ of ROS production measurement. ROS production measurement was performed by adding $100 \mu \mathrm{L}$ of ROS-Glo ${ }^{\mathrm{TM}}$ detection solution per well, before $20 \mathrm{~min}$ of incubation at $22^{\circ} \mathrm{C}$ followed by luminescence using a CLARIOstar $^{\circledR}$ microplate reader.

\section{Metabolic Flux Analysis}

For mature BMDCs (on day 10), $1.5 \times 10^{5}$ cells per well were seeded into seahorse culture plate (Agilent, 102416-100) precoated with Cell-Tak (Corning, 354240) to enable BMDCs adherence, in complete culture media supplemented with GMCSF ( $5 \mathrm{ng} / \mathrm{mL}$ ) and FLT-3L ( $25 \mathrm{ng} / \mathrm{mL}$ ). For mature BMDMs (on day 7), $0.8 \times 10^{5}$ cells per well were seeded into seahorse culture plate as described in the previous study (24). A graphical representation of the experiment design is presented in Supplementary Figure 2.

\section{Statistical Analysis}

Results are expressed as mean values \pm standard deviation (SD). Statistical analysis was performed using GraphPad Prism version 8.4.2. Data were analysed by one-way ANOVA and Tukey's multiple comparison test to analyse the difference between different groups. P-values below 0.05 were considered as significant and indicated as follows: ${ }^{\star} \mathrm{P} \leq 0.05,{ }^{*} \mathrm{P} \leq 0.01$, ${ }^{* * *} \mathrm{P} \leq 0.001$, and ${ }^{\star * * *} \mathrm{P} \leq 0.0001$ as compared with untreated cells (not exposed to NLCs).

\section{RESULTS}

\section{nNLCs and cNLCs Do Not Induce Cell Toxicity and Are Efficiently Internalised by APCs}

We first investigated whether the exposure of nNLCs and cNLCs is toxic for APCs in vitro, using a macrophage cell line (J774.1A) or primary untransformed cells extracted from bone marrow: macrophages (BMDMs) and DCs (BMDCs). Cells were exposed to nNLCs or cNLCs with concentrations ranging from 0 to $250 \mu \mathrm{g} / \mathrm{mL}$ and measured toxicity (Figure 1A). Among all the tested cells, BMDCs were most susceptible to both nNLCs and cNLCs exposure, and all the tested conditions exhibited more than $80 \%$ of cell viability. Therefore, for subsequent experiments, we chose 20 and $100 \mu \mathrm{g} / \mathrm{mL}$ as low and high standard doses, respectively, without adverse effects, that is, higher than $80 \%$ of cell viability after $24 \mathrm{~h}$ of incubation.

Next, we assayed the internalisation of both nNLCs and cNLCs by two primary cell types: BMDCs and BMDMs that are more physiologically relevant than any immune cell lines. The analysis of the time dependent engulfment of both NLCs showed that the maximum of uptake was reached after $1 \mathrm{~h}$ for both BMDCs (Figure 1B) and BMDMs (Figure 1C). Staining the cell membrane of APCs with FITC conjugated cholera toxin showed both the nanocarriers were internalised into BMDCs (Figure 1D) and BMDMs (Figure 1E) within a 24-h time frame. Therefore, from these first experiments, we can conclude that 
A
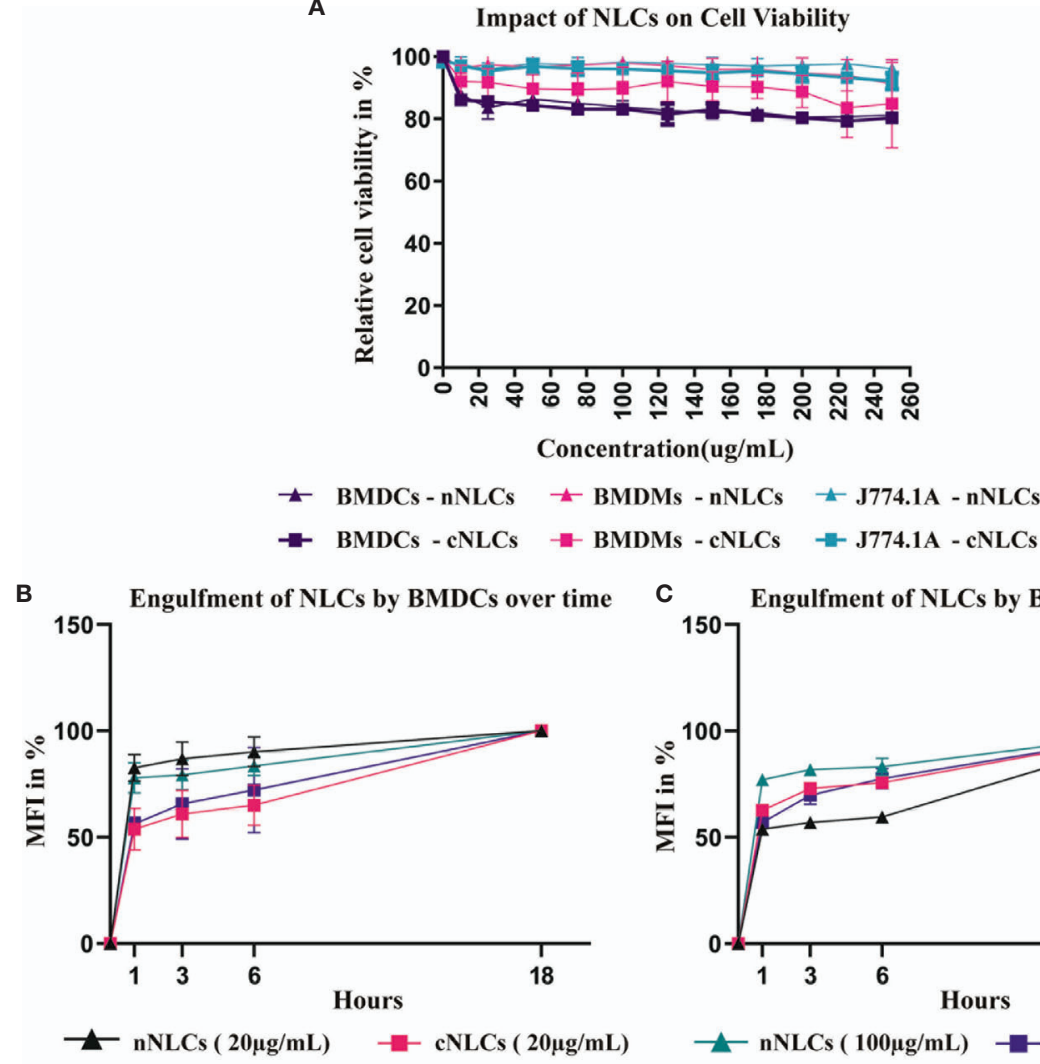

C Engulfment of NLCs by BMDMs over time

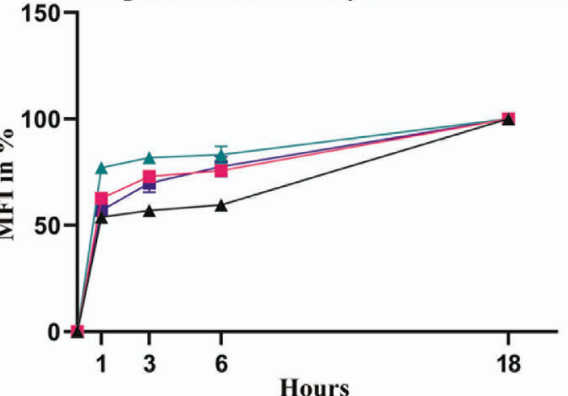

D

Dil-nNLCs + FITC-Cholera toxin Dil-cNLCs + FITC-Cholera toxin
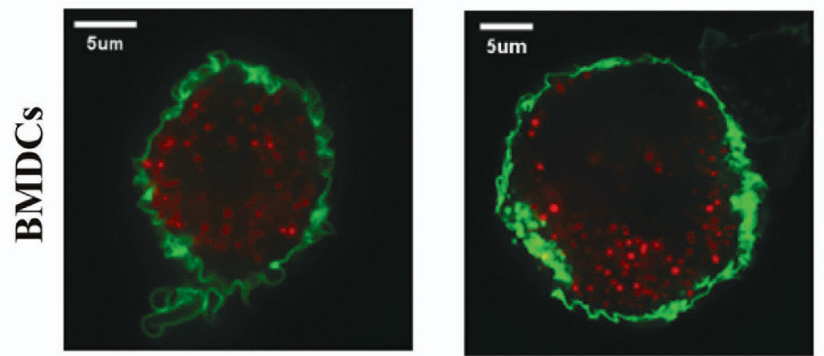

E
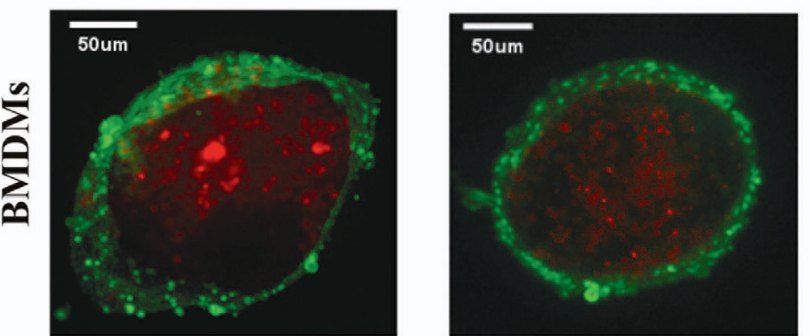

FIGURE 1 | nNLCs and cNLCs do not induce cell toxicity and are efficiently internalised by APCs (A) Cell viability (LDH release assay) of BMDCs, BMDMs and J774.1A cells was analysed after exposure to different concentrations of nNLCs and cNLCs nanocarriers for $24 \mathrm{~h}$. Data are displayed as mean \pm SD and normalised to the untreated cells ( $N=3$ independent experiments). Time-dependent engulfment of both cNLCs and nNLC in BMDCs (B) and BMDMs (C). After APCs exposure to 20 and $100 \mathrm{\mu g} / \mathrm{ml}$ of Dil labeled nNLCs or Dil labeled cNLCs nanocarriers for 1, 3, 6, $18 \mathrm{~h}$ cells were analysed by flow cytometry. Data are displayed as mean \pm SD and presented as \% of max MFI (at 18h) ( $N=3$ independent experiments). Confocal microscopy analysis of nNLCs and cNLCs uptake in (D) BMDCs and (E) BMDMs. After APCs exposure to $100 \mu \mathrm{g} / \mathrm{ml}$ of nNLCs or cNLCs nanocarriers for $24 \mathrm{~h}$, cell membranes were labelled with FITC-conjugated cholera toxin (green), and nNLCs and cNLCs are observed by excitation of Dil fluorescent dye (red). Images were acquired using a confocal spinning-disk microscope. The images displayed were representative of the majority of cells observed. 
these two nanocarriers were not toxic up to a $250-\mu \mathrm{g} / \mathrm{mL}$ concentration, while they were both efficiently internalised by APCs.

\section{nNLCs and cNLCs Are Internalised by APCs Without Affecting Their Phagocytic Capacity}

Accumulation of nanocarriers into phagocytic APCs opens the question of whether their functions could be altered, such as phagocytosis, which is one of the primary features of APCs. The phagocytic capacity of BMDCs or BMDMs was assessed by counting the number of engulfed microspheres per cell by flow cytometry. This parameter was not altered by either the neutral or the cationic nanocarrier supporting that the phagocytic capacity of both APCs was not modified by any type of nanocarrier (Figures 2A-D). Moreover, we noticed that the phagocytic capacity of BMDMs was $20 \%$ higher than that of BMDCs (Figures 2B, D).

We also verified the impact of the nanocarriers on the phagocytic capacity of J774.1A cells, a well-characterised macrophage cell line for phagocytosis analysis (25). Similarly, we did not observe a significant change in phagocytic capacity between the nanocarrier treated cells or control cells. These results obtained with the J774.1A cell line were consistent with what we observed in the primary cells (Supplementary Figures 3A, B).

\section{cNLCs but Not nNLCs Can Increase LPS Activation of BMDMs}

BMDCs were identified by CD11b and CD11c expressions (26) whereas BMDMs were marked by CD11b and F4/80 expressions (27) (see the gating strategy in Supplementary Figure 4). Activation of BMDCs and BMDMs was evaluated by the frequency of CD86 and MHC-II double-positive cells. After LPS stimulation, the frequency of $\mathrm{CD}^{+} 6^{+}$and $\mathrm{MHC}-\mathrm{II}^{+}$in BMDCs increased from $27.83 \%$ to $75.9 \%$ (Figure $\mathbf{3 A}$ and Table 2) while no significative changes were observed in BMDMs (Figure 3B).

Exposure to increasing concentrations of nNLCs or cNLCs did not significantly alter LPS-induced double expression of CD86 and MHC-II in BMDCs. In the case of unstimulated BMDMs activation, CD86 and MHC-II double-positive cell percentage was not altered when exposed to nNLCs but decreased significantly when exposed to cNLCs at the highest dose from $19.6 \%$ to $9.79 \%$. In the case of unactivated BMDMs, the percentage of CD86 positive cells remained unaltered when exposed to nNLCs (Table 2). Altogether, our data highlight that both nanocarriers do not activate BMDCs, but cNLCs slightly alter the activation of BMDMs. BMDCs, on exposure to both nanocarriers, maintained their capacity to respond to LPS activation. However, in the case of LPS-stimulated BMDMs, exposure to cNLCs significantly increased the percentage of
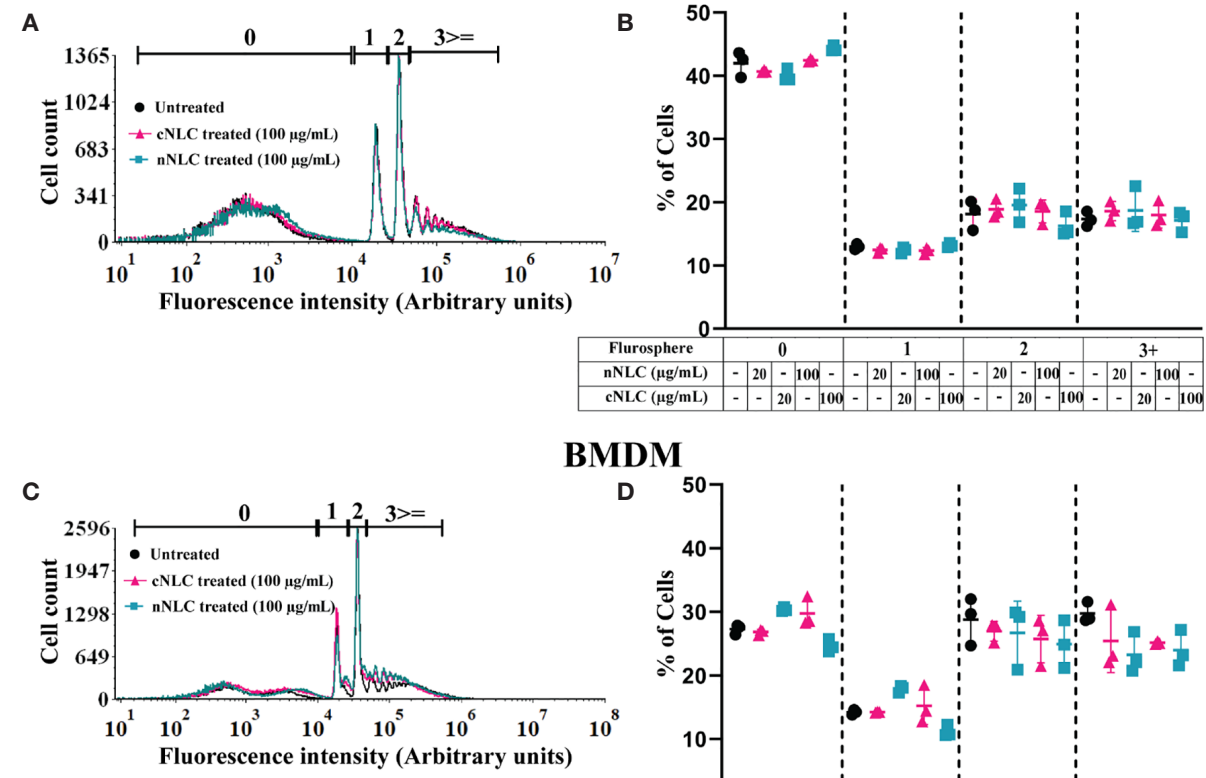

BMDM

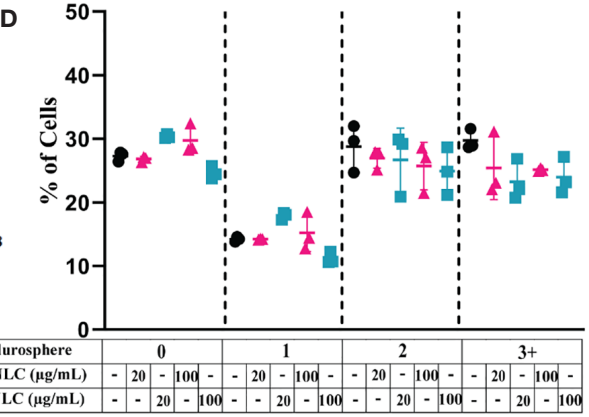

- APCs / APCs +LPS

$\triangle \mathrm{APCs}+\mathrm{nNLCs} / \mathrm{APCs}+\mathrm{nNLCs}+\mathrm{LPS} \quad \mathrm{APCs}+\mathrm{cNLCs} / \mathrm{APCs}+\mathrm{cNLCs}+\mathrm{LPS}$

FIGURE 2 | Phagocytic capacity of APCs exposed to nNLCs or cNLCs. BMDCs and BMDMs were exposed to nNLCs and cNLCs nanocarriers at 20 and $100 \mu \mathrm{g} / \mathrm{mL}$ for $24 \mathrm{~h}$, then incubated with fluorescent microspheres for $6 \mathrm{~h}$ and subsequently analysed by flow cytometry. The repartition of the cells in the 1 st, 2 nd, 3rd and 4th peak corresponds to 0, 1, 2 and 3 or more beads internalisation, respectively. Overlaid histograms are shown in (A) for BMDCs and (C) for BMDMs. The proportion of cells in each peak was analysed for (B) BMDCs and (D) BMDMs. Data are displayed as mean \pm SD ( $N=3$ independent experiments). 
A

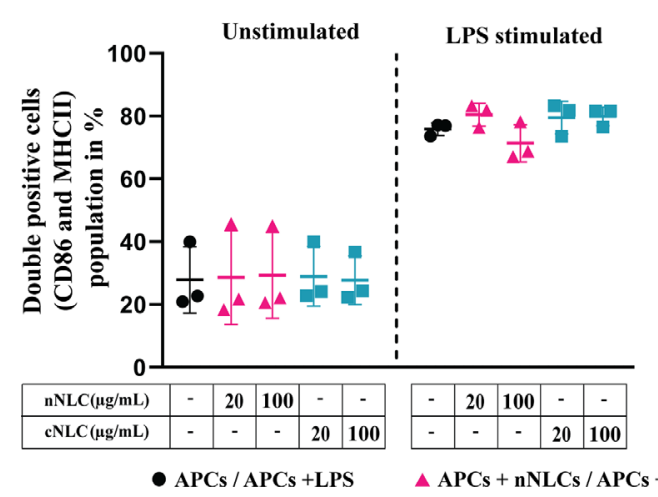

B

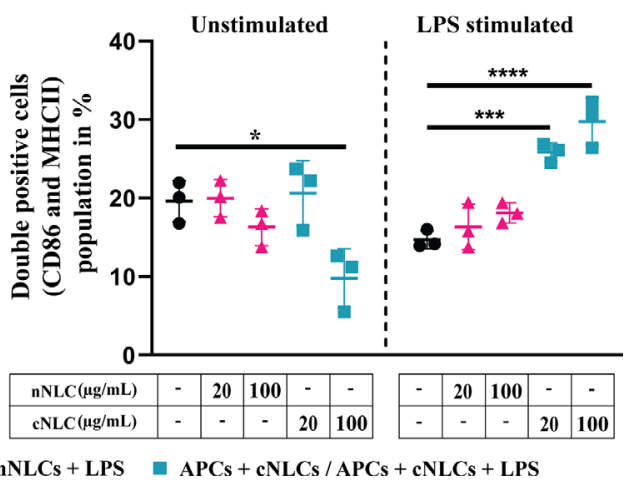

FIGURE 3 | Expression of activation surface marker in APCs following exposure to nNLCs or cNLCs. BMDCs (A) and BMDMs (B) were exposed to nNLCs or cNLCs for $24 \mathrm{~h}$, followed by LPS stimulation for an additional $24 \mathrm{~h}$. Percentage of double-positive (CD86 and MHC-II) BMDCs and CD86 positive BMDMs were determined, with gating on CD11b and Cd11c positive cells for BMDCs and CD11b and F4/80 positive cells for BMDMs. Data are displayed as mean \pm SD ( $\mathrm{N}=3$ independent experiments), and the statistical significance between nanocarrier treated or untreated groups was performed by one-way ANOVA test using Tukey's multiple comparisons test. ${ }^{*} \mathrm{P} \leq 0.05 ;{ }^{* \star *} \mathrm{P} \leq 0.001$; and ${ }^{* \star * *} \mathrm{P} \leq 0.0001$

activated BMDMs from $14.69 \%$ to $29.76 \%$, while it remained the same with the nNLCs (Figure 3B and Table 2). This suggests that exposure to nanocarriers alone is not sufficient to activate both BMDCs and BMDMs. However, in LPS-stimulated BMDMs, exposure to cNLCs increased the frequency of $\mathrm{CD}^{+} 6^{+}$and $\mathrm{MHC}$ $\mathrm{II}^{+}$activated cells. Internalisation of both lipid nanocarriers, neutral and cationic ones, is not sufficient to activate both BMDCs and BMDMs, although exposure to cNLCs enhanced the ability of BMDMs to respond to LPS stimulation.

\section{cNLCs and nNLCs Can Alter the Production of Signalling Molecules by APCs}

The capacity to produce different soluble factors, including signalling proteins such as cytokines or chemokines and other small molecular mediators such as NO and ROS, is a hallmark of APCs activation.

Having demonstrated that exposure to cNLCs could alter the activation of BMDMs in response to LPS, we wondered what would be the impact of both nanocarriers on cytokine secretion.
We observed that both nanocarriers did not induce cytokine secretion in unstimulated BMDCs and BMDMs (Figures 4A-D, left panel), except the highest dose of cNLCs but not nNLCs, which significantly increased the production of the MCP-1 chemokine in unstimulated BMDCs and to a lesser extend in unstimulated BMDMs (Figures 4E, F, left panel).

Upon LPS stimulation of APCs, nNLCs exposure did not alter IL- 6 production by both BMDCs and BMDMs. However, exposure to cNLCs significantly increased IL-6 production by BMDMs (Figure 4B, right panel) but not by BMDCs (Figure 4A, right panel). In the case of BMDCs, both nNLCs and cNLCs decreased TNF- $\alpha$ production at $100 \mu \mathrm{g} / \mathrm{mL}$ (Figure 4C, right panel). For BMDMs, TNF- $\alpha$ production was only increased at $100 \mu \mathrm{g} / \mathrm{mL}$ of $\mathrm{cNLCs}$ but not for BMDCs (Figure 4D, right panel). We also observed that treatment with cNLCs but not nNLCs significantly increased MCP-1 production in both LPSstimulated BMDCs and BMDMs (Figures 4E, F, right panel).

Two other important secretory molecules, NO and ROS productions were evaluated in the culture supernatant of APCs by Griess assay and $\mathrm{H}_{2} \mathrm{O}_{2}$ quantification, respectively. In absence

TABLE 2 | Percentage of activated APCs with or without NLCs treatment.

Double positive (CD86 and MHC-II) cells population percentage (mean \pm SD) BMDCs

BMDMs

\begin{tabular}{|c|c|c|c|c|}
\hline & & \\
\hline & Unstimulated & LPS-stimulated & Unstimulated & LPS-stimulated \\
\hline Cells & $27.83 \pm 8.58$ & $75.9 \pm 1.62$ & $19.6 \pm 2.13$ & $14.69 \pm 0.93$ \\
\hline Cells + nNLCs (20 ug/mL) & $28.61 \pm 12.22$ & $80.51 \pm 2.97$ & $19.98 \pm 1.92$ & $16.32 \pm 2.35$ \\
\hline Cells + nNLCs (100 ug/mL) & $29.3 \pm 11.21$ & $71.38 \pm 4.85$ & $16.3 \pm 1.90$ & $18.1 \pm 1.05$ \\
\hline Cells + cNLCs (20 ug/mL) & $28.97 \pm 7.79$ & $79.57 \pm 4.27$ & $20.61 \pm 3.39$ & $25.84 \pm 0.98$ \\
\hline Cells + cNLCs (100 ug/mL) & $27.74 \pm 6.37$ & $79.91 \pm 2.39$ & $9.79 \pm 3.07$ & $29.76 \pm 2.45$ \\
\hline
\end{tabular}

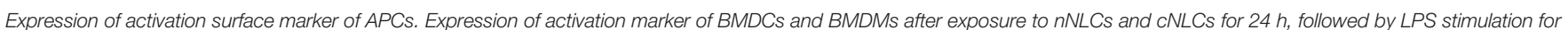

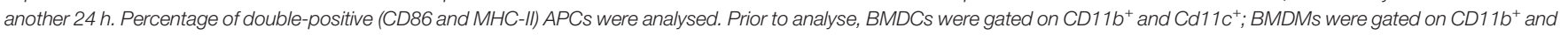
$\mathrm{F} 4 / 8 \mathrm{O}^{+}$; and the data are presented in tabular form. Results are mean $\pm S D$ of 3 independent experiments. 
A

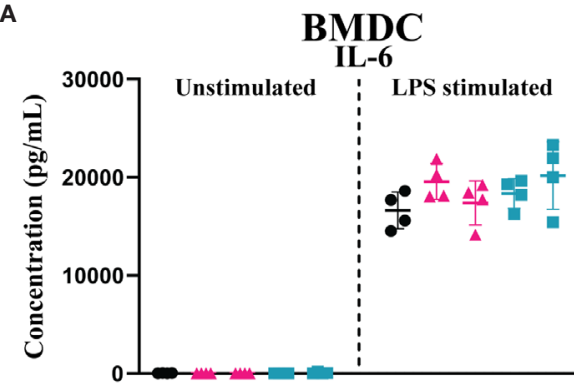

C

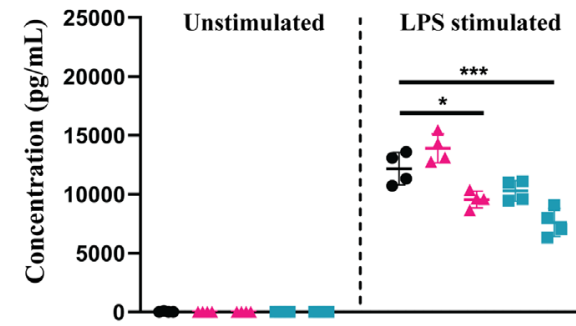

E

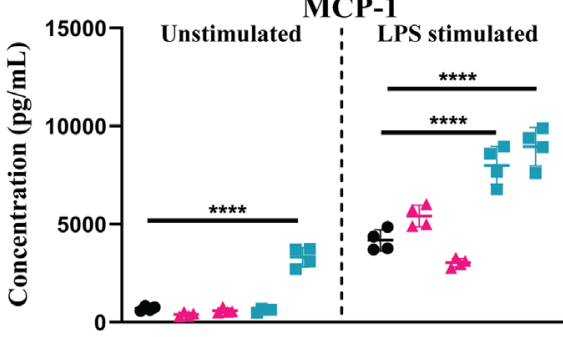

G

U⿺辶ِ
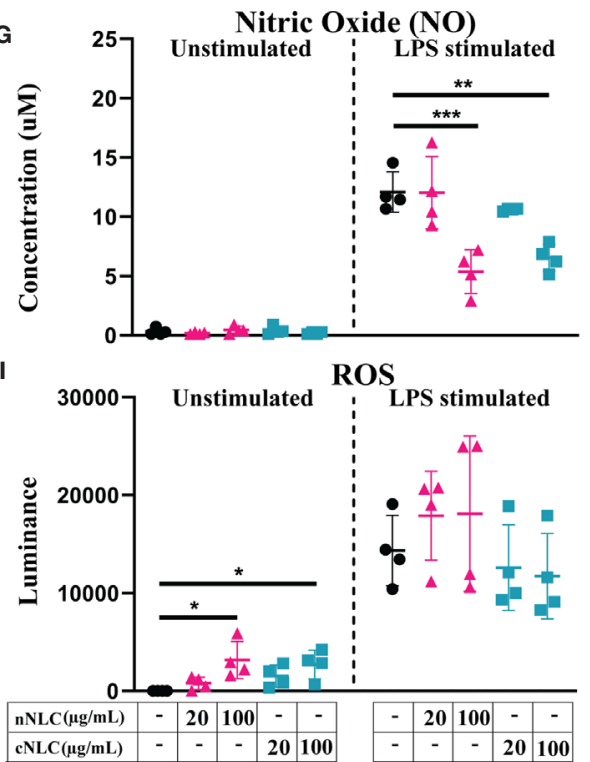

B

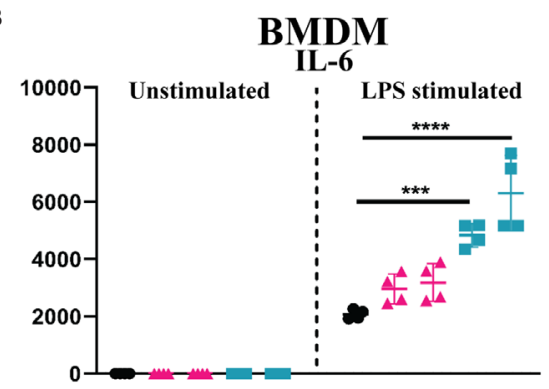

D

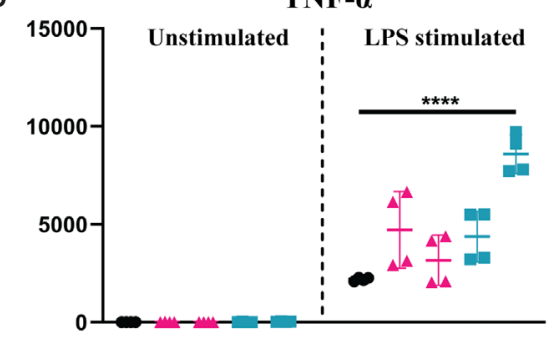

F

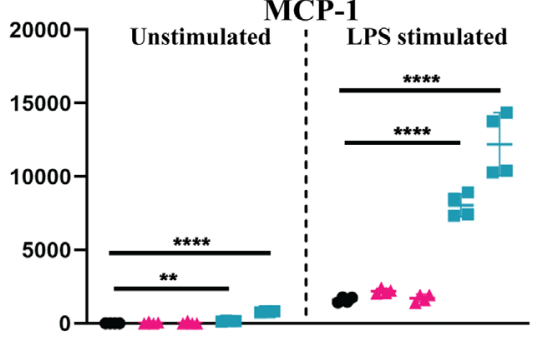

H

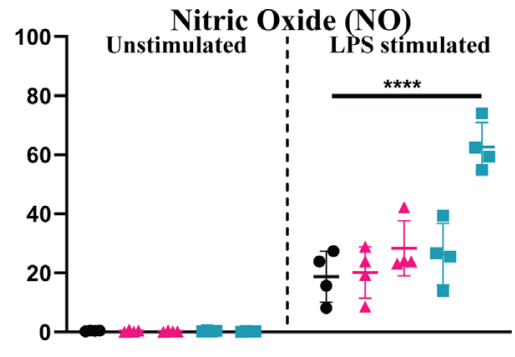

$J$

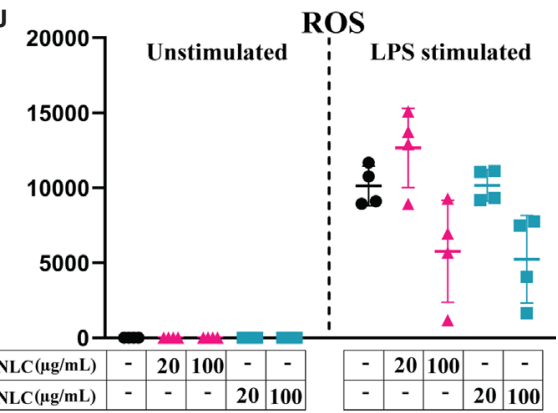

FIGURE 4 | Secretions of signalling factors by APCs in response to nNLCs or cNLCs. Relative cytokine and chemokine concentration in the supernatant of BMDCs and BMDMs exposed to nNLCs or CNLCs and activated or not by LPS was determined by immunoassay. Secretion of the IL-6 cytokine in (A) BMDCs and (B) BMDMs; the TNF $\alpha$ cytokine in (C) BMDCs and (D) BMDMs and the chemokine MCP-1 in (E) BMDCs and (F) BMDMs. Relative NO concentration in the supernatant of BMDCs (G) and BMDMs (H) cells exposed to nNLCs or CNLCs and activated or not by LPS was determined by Griess assay. ROS production by BMDCs (I) and BMDMs (J) cells exposed to nNLCs or CNLCs and activated or not by LPS was determined by ROS-Glo ${ }^{\mathrm{TM}} \mathrm{H}_{2} \mathrm{O}_{2}$ assay. Data are displayed as mean $\pm \mathrm{SD}(\mathrm{N}=4$ independent experiments), and the statistical significance between nanocarrier treated or untreated groups was performed by one-way ANOVA test using Tukey's multiple comparisons test. ${ }^{*} \mathrm{P} \leq 0.05 ;{ }^{* *} \mathrm{P} \leq 0.01 ;{ }^{* \star *} \mathrm{P} \leq 0.001$; and ${ }^{* \star *} \mathrm{P} \leq 0.0001$. 
of LPS stimulation, we did not observe a production of NO by BMDCs and BMDMs in response to both nanocarriers (Figures 4G, H, left panel) although ROS production was detected by BMDCs treated with $100 \mu \mathrm{g} / \mathrm{mL}$ of either nNLCs or cNLCs but not in BMDMs (Figures 4I, J, left panel). In LPSstimulated conditions, both nNLCs and cNLCs at highest dose decreased NO production by BMDCs (Figure 4G, right panel), while the only cNLCs were responsible for increasing NO production in BMDMs (Figure $\mathbf{4 H}$, right panel). After stimulation by LPS, both APCs produced increased quantities of ROS, but its production was not significantly altered by exposure to both nanocarriers (Figures 4I, J, right panel). These results indicate that BMDCs and BMDMs are differently affected by neutral or cationic nanocarriers regarding their capacity to produce $\mathrm{NO}$ and ROS and depending on activation stimuli.

Overall, nNLCs have only limited influence on the productions of signalling molecules, whereas cNLCs display significant effects, especially for inflammatory signals. The influence of cNLCs is clearly demonstrated in activated BMDMs by the increases of IL- 6 , TNF- $\alpha$, MCP-1 secretions and NO production. Both nNLCs and cNLCs share most of their features such as their same size and composition; therefore, their major difference resides in their surface charge. This led us to hypothesise that this difference in the surface charge may be responsible for different effects driven by these two nanoparticles on APCs.

\section{nNLCs and cNLCs Have a Significant Impact on the Mitochondrial Metabolism of BMDMs but Not on That of BMDCs}

As cellular metabolism plays a key role in different functions of APCs, we sought to determine the effect of differentially charged LNCs on mitochondrial metabolism. For instance, proinflammatory stimuli by LPS are known to trigger a metabolic switch that would enhance glycolysis, whereas enhanced FAO and mitochondrial OXPHOS are hallmarks of IL-4-induced antiinflammatory activity in immune cells.

Upon exposition to both nanocarriers, no alteration in the basal respiration, maximal respiration capacity, spare respiratory capacity, nonmitochondrial oxygen consumption and coupling efficiency (Supplementary Figures 5A, C, E and Figures 6A, C), proton leak or ATP production (Figures $\mathbf{5 A}, \mathbf{C}$ ) were found in unstimulated or stimulated BMDCs.

In BMDMs, exposure to both nanocarriers increased basal respiration and nonmitochondrial oxygen consumption of unstimulated cells at $100 \mu \mathrm{g} / \mathrm{mL}$, as well as the nonmitochondrial oxygen consumption of LPS-stimulated cells treated with the nNLCs (Supplementary Figures 5B and 6B). Treatment with $100 \mu \mathrm{g} / \mathrm{mL}$ of cNLCs significantly increased the proton leak, Adenosine triphosphate (ATP) production, basal respiration, maximal respiration capacity, spare respiratory capacity and nonmitochondrial oxygen consumption (Figures 5B, D and Supplementary Figures 4B, D, F, and 5B) in unstimulated or IL-4-stimulated BMDMs whereas the nNLCs did only slightly increase basal respiration and nonmitochondrial oxygen consumption (Supplementary Figures 5A and 6A).
It is to be noted that both nanocarriers did not impair the coupling efficiency of unstimulated or stimulated BMDMs (Supplementary Figure 6B).

As a whole, our results demonstrate that the cNLCs have a more important effect on BMDMs' metabolism compared with the nNLCs, while both nanocarriers have little effect on the metabolism of BMDCs.

\section{nNLCs and cNLCs Alter the Glycolysis of BMDMs and Not of BMDCs}

Considering the alterations of the mitochondrial metabolism induced by the cNLCs and to a lesser extent the nNLCs, we sought to investigate their effects on the glycolytic profile of APCs as LPS-stimulated cells are mostly dependent on glycolysis. To evaluate the different glycolytic parameters of BMDCs and BMDMs, cells were first pretreated with different concentrations of both nanocarriers and then stimulated with LPS or IL-4 for $24 \mathrm{~h}$. After stimulation, the extracellular acidification rate (ECAR) was measured using the glyco stress assay.

Unlike for BMDCs that did not show any alteration in glycolysis (Figure 5E) or glycolytic capacity (Supplementary Figure 7A), BMDMs' glycolysis (Figure 5F) and glycolytic capacities (Supplementary Figure 7B) were increased in both unstimulated and stimulated conditions when exposed to $100 \mu \mathrm{g} / \mathrm{mL}$ of cNLCs. However, exposure to nNLCs did not induce any alteration in glycolysis or glycolytic capacity in BMDMs regardless of stimulating conditions (Figure 5F and Supplementary Figure 7B).

The combination of these results reveals that the cationic but not the nNLCs at the highest concentration alter the glycolytic profile in BMDMs. Conversely, both nanocarriers have no effect on glycolysis in BMDCs.

\section{Reversing the Surface Charge With a Nucleic Acid Cargo Prevents Adverse Effects of cNLCs on APCs}

As previous experiments have pointed out, at $100 \mu \mathrm{g} / \mathrm{mL}$, cNLCs had a more dramatic effect on BMDMs' physiology than nNLCs; we wondered whether the surface charge could explain the differences observed.

This led us to investigate whether we could reverse the phenotype observed on APCs by reversing the surface charge of the cNLCs with a nucleic acid cargo, here a negative control siRNA (siMock). We used different surface charges by finetuning the ratio of the positively charged amine groups of cNLCs nanocarriers ( $\mathrm{N}=\mathrm{NH}^{3+}$ group) relative to the negatively charged phosphate groups (P) from each phosphodiester bonds within the nucleic acid sequence, hence called N/P ratio. After complexation between siRNA and cNLCs nanocarriers, the zeta potential and hydrodynamic diameter of these nanocomplexes were measured. Naked cNLCs showed a zeta potential of $45.80 \pm 3.8 \mathrm{mV}$ in $1 \mathrm{mM} \mathrm{NaCl}$ while increasing amounts of the nucleic acid cargo and thus decreasing the N/P ratio lead to lower the zeta potential values down to $-9.97 \pm 0.94$ $\mathrm{mV}$, while naked nNLCs was measured at $-16.50 \pm 0.53 \mathrm{mV}$ (Figure 6A). It is to be noted that the complexation of cNLCs with different quantities of siRNA did not significantly alter the size of the nanocomplexes (Figure 6B). 
A

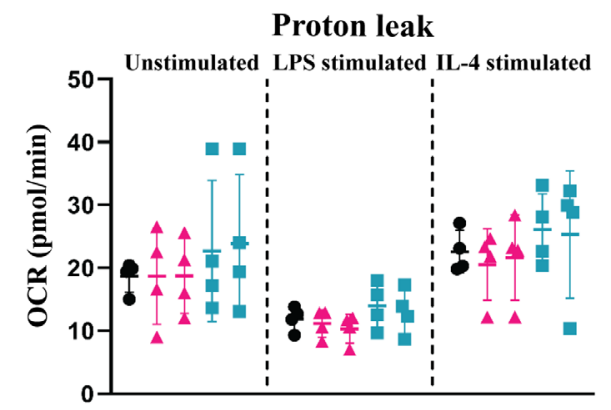

C

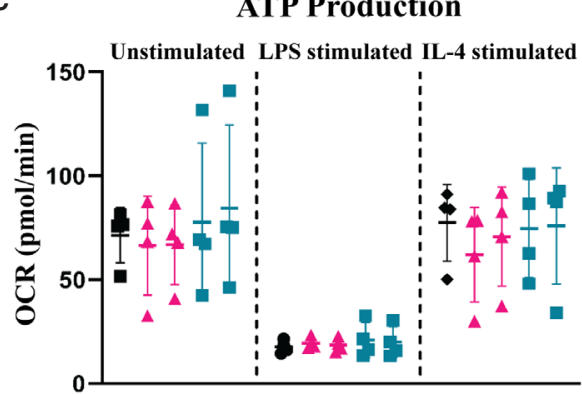

E
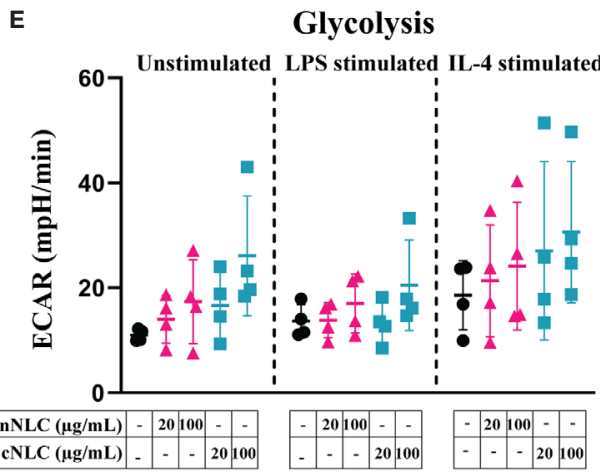

B

BMDM

Proton leak

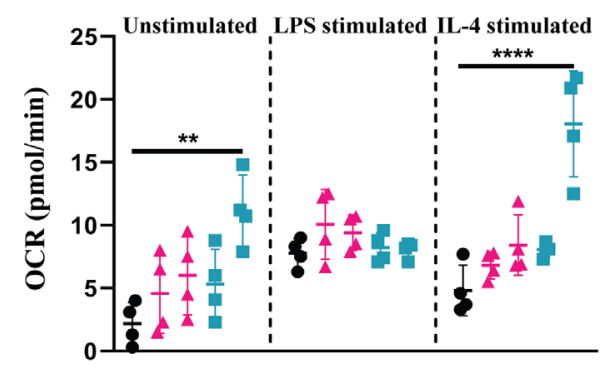

D

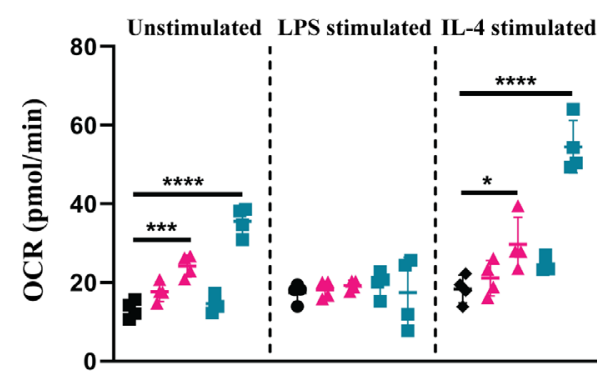

F

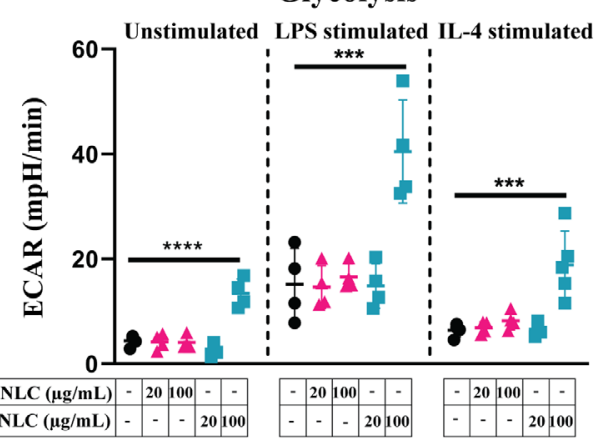

cNLC $(\mu \mathrm{g} / \mathrm{mL})$ -

- APCs / APCs +LPS

$\triangle \mathrm{APCs}+\mathrm{nNLCs} / \mathrm{AP}$

FIGURE 5 | Mitochondrial metabolism in naïve, classically activated or alternatively activated APCs in response to nNLCs or cNLCs. (A, B) Proton leak, (C, D) ATP production and (E, F) glycolysis in BMDCs and BMDMs, respectively, were measured after exposure to cNLCs or nNLCs for 24 h and activated by LPS or IL-4 for another $24 \mathrm{~h}$. Oxygen consumption rate (OCR) and ECAR were quantified using a seahorse XF analyser. Data were normalised by cell number based on cell count (Hoechst 33342 staining) and are displayed as mean $\pm \mathrm{SD}$ ( $\mathrm{N}=4$ independent experiments). The statistical significance between nanocarrier treated or untreated groups was performed by one-way ANOVA test using Tukey's multiple comparisons test. ${ }^{\star} \mathrm{P} \leq 0.05 ;{ }^{\star \star} \mathrm{P} \leq 0.01$; ${ }^{\star \star \star} \mathrm{P} \leq 0.001 ;$ and ${ }^{\star \star \star \star} \mathrm{P} \leq 0.0001$.

Using different $\mathrm{N} / \mathrm{P}$ ratios, we generated nanocarriers with different zeta potentials that we subsequently used to investigate their effects on BMDMs functions. An experimental design of metabolic flux analysis for reversal of nanocarrier surface charge is depicted in Supplementary Figure 8. BMDMs were exposed to 100 $\mu \mathrm{g} / \mathrm{mL}$ of cNLCs nanocarrier, cNLCs-siRNA nanocomplexes at N/P 8 to N/P 1 or nNLCs nanocarrier. The culture supernatants were collected, and the secretion of pro-inflammatory cytokines (IL-6, TNF $\alpha$ ) or chemokine (MCP-1) was quantified by immunoassay. IL-6 and TNF $\alpha$ productions by LPS-stimulated BMDMs were correlated to the zeta potential of the nanocarriers (Figures 6C, D), that is, the productions were maximum with cNLCs and decreased when cNLCs are complexed to siRNA reaching at $\mathrm{N} / \mathrm{P}$ ratio 1 a similar level than the one obtained with nNLCs. The production of NO and MCP-1 by LPS-activated BMDMs also decreased with lower N/P ratios but to a lesser extent than for IL-6 and TNFo (Figure 6E and Supplementary Figure 9A).

To analyse the effect of the surface charge on glycolysis, we measured ECAR in BMDMs exposed to nanocomplexes at different $\mathrm{N} / \mathrm{P}$ ratios and then stimulated or not with LPS. Both unstimulated and LPS-stimulated BMDMs showed a decrease in both glycolysis and glycolytic capacities with decreasing zeta potential and almost down to the same values as that of the nNLCs for the unstimulated cells (Figure 6F and Supplementary Figure 9C).

Next, we analysed the effect of the surface charge on the mitochondrial metabolism of BMDMs, by measuring the OCR in 
A

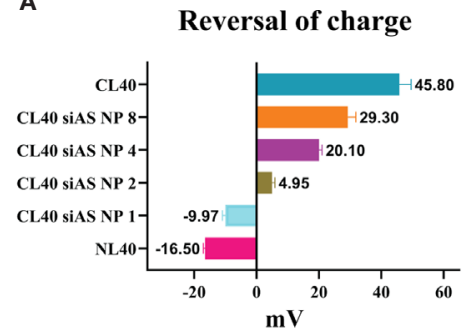

C

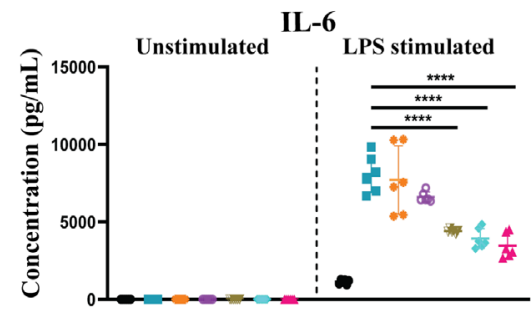

E

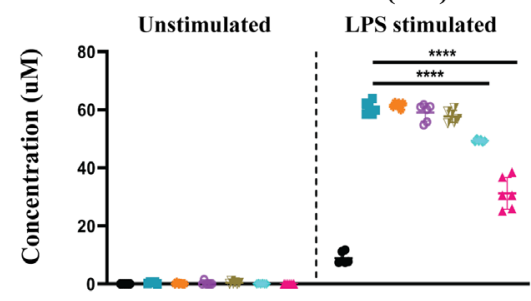

G

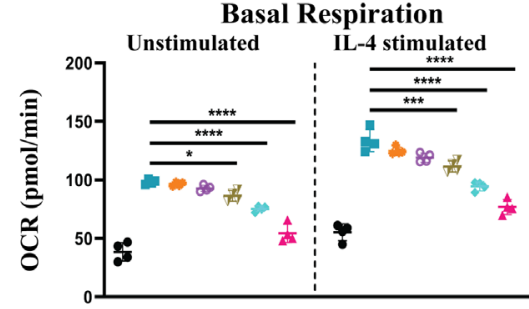

I

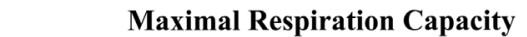

Maximal Respiration Capacity

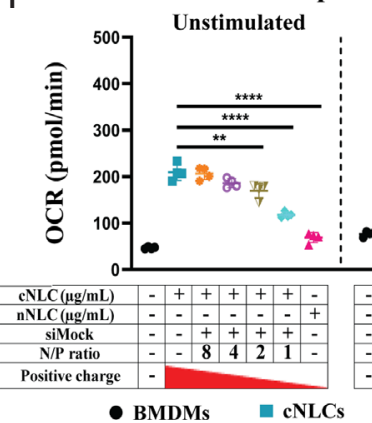

IL-4 stimulated

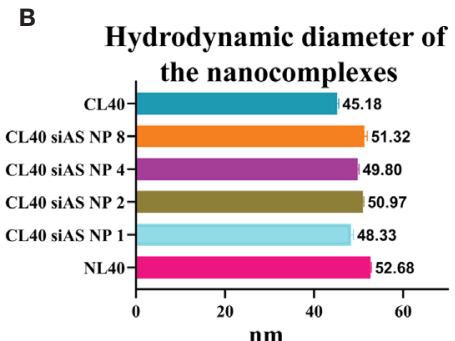

D

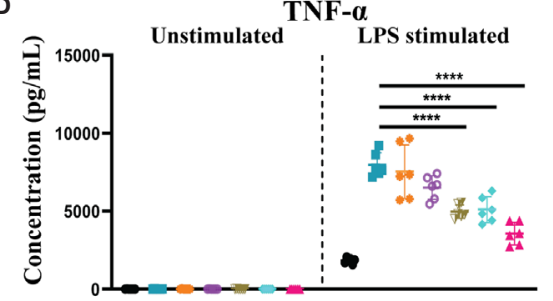

Glycolysis

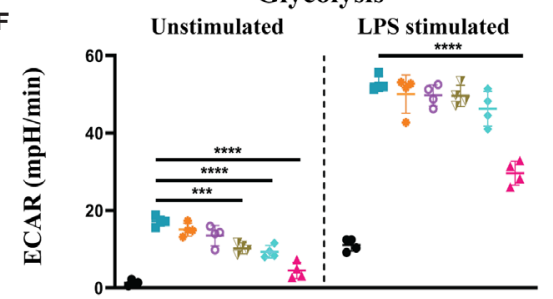

H

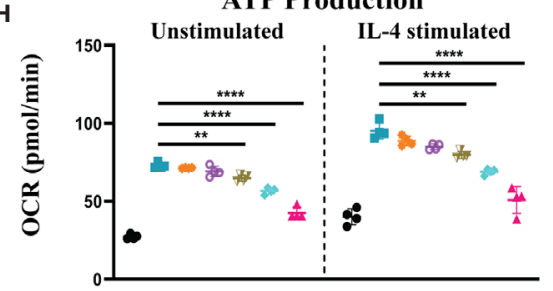

$\mathbf{J}$

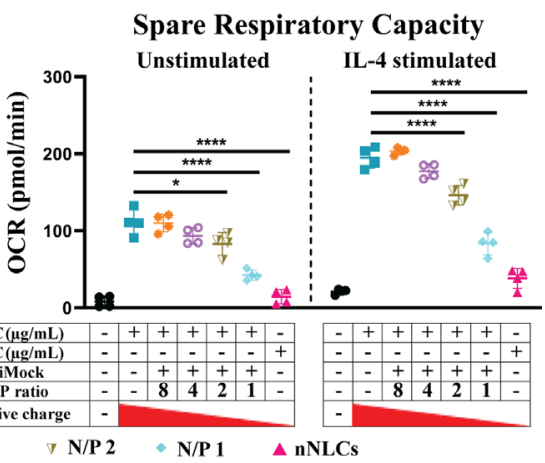

FIGURE 6 | Reversing the surface charge with a nucleic acid cargo prevent adverse effects of cNLCs on APCs. (A) The zeta potential measurement of cNLCs complexes with siRNA at different N/P ratios was performed on a zetasizer instrument by ELS in 1 mM NaCl. (B) The hydrodynamic diameter of cNLCs complexes with siRNA at different N/P ratios was measured on a zetasizer instrument by DLS in PBS buffer. (C) IL-6 and (D) TNF $\alpha$ secretion was quantified from the supernatant of BMDMs exposed to $100 \mu \mathrm{g} / \mathrm{mL}$ of cNLCs complexes with siRNA at different N/P ratios and activated or not by LPS. (E) NO concentration in the supernatant of BMDMs exposed to $100 \mu \mathrm{g} / \mathrm{mL}$ of cNLCs complexes with siRNA at different N/P ratios and activated or not by LPS was determined by Griess assay. (F) Glycolysis in BMDMs exposed to $100 \mu \mathrm{g} / \mathrm{mL}$ of cNLCs complexes with siRNA at different N/P ratios and activated or not by LPS was determined by ECAR.

(G) Basal respiration, (H) ATP production, (I) maximal respiration capacity and $\mathbf{( J )}$ spare respiratory capacity in BMDMs exposed to $100 \mu \mathrm{gg} / \mathrm{mL}$ of $\mathrm{cNLCs}$ alone or complexes with siRNA at different N/P ratios and activated or not by IL-4 was determined by OCR. OCR and ECAR were quantified using a seahorse XF analyser. Data were normalised by cell number based on cell count (Hoechst 33342 staining) and are displayed as mean \pm SD ( $N=4$ or 6 independent experiments). The statistical significance between nanocarrier treated or untreated groups was performed by one-way ANOVA test using Tukey's multiple comparisons test. * $\mathrm{P} \leq 0.05$; ${ }^{\star \star} \mathrm{P} \leq 0.01 ;{ }^{\star \star \star} \mathrm{P} \leq 0.001 ;$ and ${ }^{\star \star \star \star} \mathrm{P} \leq 0.0001$. 
BMDMs exposed to nanocomplexes at different $\mathrm{N} / \mathrm{P}$ ratios and then stimulated or not with IL-4. The exposure to differently charged nanocarriers showed a decrease in basal respiration, maximal respiration capacity, ATP production, spare respiratory capacity and proton leak correlated with a decrease in zeta potential in both unstimulated and IL-4-stimulated BMDMs (Figures 6G-J and Supplementary Figure 8B). However, the effect of differently charged nanocarriers on both unstimulated and IL-4-stimulated BMDMs was not statistically significant for nonmitochondrial oxygen consumption and percentage of coupling efficiency (Supplementary Figures 9D, E).

Altogether, these results revealed that decreasing zeta potential, hence the surface charge of the cNLCs, was able to reverse their effect on the different cellular functions of primary BMDMs upon both pro- and anti-inflammatory stimulations. Moreover, using a range of N/P ratios representing the surface charge of the nanocarriers, we demonstrated that the alteration of the BMDMs physiology was proportional to the overall net surface charge of nucleic acid-loaded LNPs.

\section{DISCUSSION}

Lipid-based nanocarriers are promising delivery systems for imaging (28), gene therapy including nucleic acids delivery (29) such as siRNA transfection $(13,30)$ or mRNA vaccine delivery (31), drug delivery (32), adjuvant delivery system (33) and other biomedical applications.

Nanoparticles composed of cationic lipids have a strong capacity for binding and condensing nucleic acid by electrostatic interactions at the level of the phospholipid layer and deliver the payload across cellular membranes within the target cell cytoplasm (34). However, when designing a lipidbased nanocarrier, the composition of the lipids defines the protein corona around the nanocarrier that is closely linked with the activation of the immune system leading to undesired side effects and biodistribution $(35,36)$. It is well known that different components of lipid-based carriers such as DOPE and DOTAP facilitate the formation of protein corona eventually causing undesired side effects (37). One of the most efficient ways to reduce the nanocarrier-protein interaction and formation of protein corona is wrapping the nanocarrier with linear chains of PEG (38). PEGylation acts not only as an anti-opsonisation strategy but also as a thermodynamic shield that reduces nonspecific protein adsorption $(39,40)$. As our cNLCs contain DOPE and DOTAP, they were covered with $2 \mathrm{kDa}$ PEG chains to limit the adsorption of proteins and direct interaction with plasma membrane as shown in a previous study (41), although preserving their capacity of the complexation with nucleic acids. However, it remains to assess the effects of cNLCs on different immune cells to precisely manage their future uses.

To understand the effect of differently charged NLCs, we opted for ex vivo experiments as an alternative to in vivo experiments, allowing for more regulated manipulation of cell functions and processes. Although cell lines have played a crucial role in scientific progress for decades, researchers are now increasingly skeptical when interpreting data generated from cell lines only. Factors such as misrepresented and contaminated cell lines have triggered a strong interest in primary cells $(42,43)$. In our study, to be closer to the physiological conditions, we conducted our experiments on BMDMs and BMDCs. Based on the results presented here, in unstimulated BMDCs and BMDMs, NLCs had very few effects on the cellular production of soluble factors. Interestingly, after LPS stimulation, macrophages and DCs responded differently when treated with cNLCs and nNLCs. In the case of BMDMs, after LPS stimulation, cNLCs at high concentration provoked an enhanced immune response by increasing the production of different secretory proinflammatory molecules including IL-6, TNF- $\alpha$, and MCP-1, while nNLCs did not. However, in the case of BMDCs, we observe a reduction in TNF- $\alpha$ secretion by nNLCs and cNLCs exposed LPS-stimulated. Under LPS stimulation, cNLC-exposed BMDCs and BMDMs increase their production of MCP-1. MCP-1 is one of the essential chemokines that governs the migration and infiltration of monocyte and macrophage (44). Elevations of MCP-1 production have been reported after the exposure of several nanomaterials such as gold NPs on BMDMs and BMDCs (24) or nickel NPs on mesothelial cells (45). Hence, MCP-1 may be considered as a sensitive indicator of NPs exposure. MCP-1 is known to be associated with some inflammatory chronic diseases such as rheumatoid arthritis (46) or allergic asthma development (47). Therefore, it is important to consider the MCP-1 level when using cNLCs in vivo administration that might facilitate the emigration of immature myeloid cells at the site of exposure and promote inflammation.

To assess the influence of NLCs on the metabolism of BMDMs and BMDCs, we polarised these cells with either LPS or IL-4. While LPS-activated pro-inflammatory cells undergo a metabolic switch to enhanced glycolysis $(48,49)$, IL-4 induces alternatively activated cells towards an anti-inflammatory response, which would then rely mostly on FAO and mitochondrial OXPHOS (50). As a result, altered metabolism is not only a characteristic of macrophage cell functions but also a prerequisite for a proper response to an immune stimulus. We demonstrated that both NLCs did not alter the basal mitochondrial respiration of BMDCs. However, in the case of BMDMs, basal respiration increased when exposed to the highest concentration used with both NLCs, indicating that the concentration of either neutral or cationic cargo must be finely determined. While no metabolic change was observed in BMDCs, they showed an increase of glycolysis and mitochondrial respiration specific of positive cNLCs. A previous study has shown a positive association between the glycolytic and the secretory activities in macrophages; however, the same was evaluated under LPS stimulation (49). In unstimulated conditions with cNLCs exposure, we did not observe this coupling, probably because the cNLCs-induced increase of glycolysis is not high enough to drive secretory adaptations as observed in cNLCstreated BMDMs under LPS stimulation. It is noteworthy that LPSactivated BMDMs rely on mitochondrial respiration. Based on these results obtained in vitro, we can assume that positive charge of cNLCs in vivo would not significantly affect the basal level of unstimulated DCs or macrophages secretory activity, hence preventing 
unintended immune responses (suppression or activation) and subsequent harmful outcomes (cancer or autoimmunity).

For our investigations, we used two NLCs with similar composition and size but solely differing by their zeta potentials. Therefore, the effects on the cellular functions of APCs observed only with cNLCs may be linked to their respective charge. This could be explained by three hypotheses: 1) the lipid composition of the NLCs $(35), 2)$ the net surface charge of NLCs (51) and 3) the protein corona around NLCs (37, 52). Previous studies showed that solid lipid NLCs were efficiently phagocytosed by macrophages but cationic NLCs led to increased cytotoxic effects than neutral equivalents supporting the influence of the charge on APC fate (53). An example of the effect of the charge on the activation of DCs was provided in the study of cationic hydrogel rod-shaped NLCs, which were more efficient than anionic equivalent NLCs in inducing specific immune responses (54). However, influence of cationic charge of NLCs on DC activation is not universal; for instance, both some cationic PLGA-based NLCs did not provoke significant activation of BMDCs from mice (55). Other parameters like the nature of the components of the NLCs must be considered such as DOTAP which induces pro-inflammatory effect (56) and used in lipid NLCs as adjuvant for vaccine purpose (57). Altogether, these studies highlight the difficulty in defining general rules in the reactivity of APCs after exposure to NLCs that results from the huge variety of NLCs, the diversity of APCs and the great sensitivity of APCs to their environment.

Here, we demonstrate that reversing the net charge of positively charged lipid NLCs by complexing with negatively charged RNA, can reverse the effect of charged carriers on different cellular functions. For this, we studied the effect of the charge of the nanocarrier using BMDMs as a cellular model since they appeared to be the most affected cells by the exposure to cNLCs. By modifying the net surface charge of the cNLCs using siRNA at different N/P ratios, we observed that the increase of the production of pro-inflammatory secretory molecules (IL-6, TNF- $\alpha$, MCP-1 and NO) was proportional to the net surface charge of the lipid nanocarriers. In parallel, metabolic parameters, including basal respiration, maximal respiration capacity, ATP production, spare respiratory capacity and proton leak, were also modulated accordingly to the charge of the lipid nanocarriers. These results show that the effects of positively charged nanocarriers, such as cNLCs, can be reversed by the complexation of negatively charged ligands, such as RNA, proportionally to the net charge of the resulting nanocarrier. Different applications could then be developed with cNLCs associated with RNA, including RNAi therapeutics as well as mRNA delivery for vaccinal purposes, even in the context of immune disorders.

Several studies reported some effects of the charge of nanoparticles on cell behaviour. For instance, N-Arginine-Noctyl chitosan is used to synthesise $\mathrm{pH}$-sensitive charge-reversal lysosomolytic nanocarriers, which could reduce the potential toxicity of the nanocarrier as well as increase the drug delivery efficiency (58). Moreover, it has been shown that that chargereversal nanocarriers enhanced gene delivery to the tumor site
(59). Furthermore, researchers demonstrated that the use of chitosan and the $\mathrm{pH}$-responsive charge-reversible polymer enhanced the siRNA delivery (60). Here, our results highlight that fine-tuning of the surface charge of cationic NLCs with an oppositely charged biomaterial, for instance, nucleic acid, could prevent immunostimulation properties of the cationic carrier and has to be kept in mind for the future use of such carriers for therapeutic applications. Overall, using the same cationic lipid nanocarrier with tunable surface charge, we propose that positive charge is one of the major factors responsible for the alteration of the immune response.

\section{CONCLUSION}

In conclusion, both BMDCs and BMDMs responded differently when exposed to the cationic or neutral variation of the same lipid nanocarriers. Therefore, it is highly relevant to include both cell types in the case of immunotoxicity analysis. We demonstrated that both nanocarriers, at low concentration, did not significantly alter several functions of both APCs. However, the cationic nanocarrier, at the highest concentration, induced alterations of some functions of APCs. We demonstrated that this effect on APCs was dependent on the net positive charge surface charge of the lipid carrier that could be offset by loading nucleic acid cargo that mediated reversal of the charge. Finally, we propose that tuning the nucleic acid load, hence, the surface charge of NLCs is critical to their use for therapy and prevent the alteration of immune cell response to stimuli.

\section{DATA AVAILABILITY STATEMENT}

The raw data supporting the conclusions of this article will be made available by the authors, without undue reservation.

\section{AUTHOR CONTRIBUTIONS}

$\mathrm{AD}, \mathrm{AN}, \mathrm{FC}, \mathrm{CF}, \mathrm{FN}$, and PM wrote the manuscript. AN, DJ, $\mathrm{ME}$, and FN synthesized the nanoparticles and performed their physico-chemical characterization. $\mathrm{AD}, \mathrm{CF}$, and $\mathrm{PM}$ designed and performed cell experiments. FC, EJ-M, FN, and PM analysed the data and reviewed the study. All authors contributed to the article and approved the submitted version.

\section{FUNDING}

This work was supported by INSERM and CEA. This project has received funding from the European Union's Horizon 2020 research and innovation program H2020 "NEWDEAL" (grant agreement No. 720905). AD, AN, and FC were supported by a fellowship from H2020 NEWDEAL project.

\section{ACKNOWLEDGMENTS}

The authors acknowledge the staff of the animal facility of IAB, C. Charrat for technical support, M. Pezet for confocal 
and flow cytometry analysis, S. Blanchet for her expertise in SeaHorse analysis, Z. Macek-Jilkova for stimulating discussions. This publication reflects only the author's view and the Commission is not responsible for any use that may be made of the information it contains.

\section{REFERENCES}

1. Yousefi N, Tufenkji N. Probing the Interaction Between Nanoparticles and Lipid Membranes by Quartz Crystal Microbalance With Dissipation Monitoring. Front Chem (2016) 4:46. doi: 10.3389/fchem.2016.00046

2. Stoddard BL, Khvorova A, Corey DR, Dynan WS, Fox KR. Editorial: Nucleic Acids Research and Nucleic Acid Therapeutics. Nucleic Acids Res (2018) 46 (4):1563-4. doi: 10.1093/nar/gky059

3. Xue HY, Guo P, Wen WC, Wong HL. Lipid-Based Nanocarriers for RNA Delivery. Curr Pharm Des (2015) 21(22):3140-7. doi: 10.2174/1381612821666150531164540

4. Chira S, Jackson CS, Oprea I, Ozturk F, Pepper MS, Diaconu I, et al. Progresses Towards Safe and Efficient Gene Therapy Vectors. Oncotarget (2015) 6(31):30675-703. doi: 10.18632/oncotarget.5169

5. Hu B, Zhong L, Weng Y, Peng L, Huang Y, Zhao Y, et al. Therapeutic siRNA: State of the Art. Signal Transduction Targeted Ther (2020) 5(1):101. doi: 10.1038/s41392-020-0207-x

6. Mehnert W, Mäder K. Solid Lipid Nanoparticles: Production, Characterization and Applications. Advanced Drug Deliv Rev (2001) 47 (2):165-96. doi: 10.1016/S0169-409X(01)00105-3

7. zur Mühlen A, Schwarz C, Mehnert W. Solid Lipid Nanoparticles (SLN) for Controlled Drug Delivery - Drug Release and Release Mechanism. Eur J Pharmaceutics Biopharmaceutics (1998) 45(2):149-55. doi: 10.1016/S09396411(97)00150-1

8. Müller RH, Radtke M, Wissing SA. Solid Lipid Nanoparticles (SLN) and Nanostructured Lipid Carriers (NLC) in Cosmetic and Dermatological Preparations. Adv Drug Deliv Rev (2002) 54 Suppl 1:S131-55. doi: 10.1016/ S0169-409X(02)00118-7

9. Kim HR, Kim IK, Bae KH, Lee SH, Lee Y, Park TG. Cationic Solid Lipid Nanoparticles Reconstituted From Low Density Lipoprotein Components for Delivery of siRNA. Mol Pharmaceutics (2008) 5(4):622-31. doi: 10.1021/ mp8000233

10. del Pozo-Rodríguez A, Delgado D, Solinís MA, Gascón AR, Pedraz JL. Solid Lipid Nanoparticles: Formulation Factors Affecting Cell Transfection Capacity. Int $J$ Pharmaceutics (2007) 339(1):261-8. doi: 10.1016/j.jipharm.2007.03.015

11. Taratula O, Kuzmov A, Shah M, Garbuzenko OB, Minko T. Nanostructured Lipid Carriers as Multifunctional Nanomedicine Platform for Pulmonary CoDelivery of Anticancer Drugs and siRNA. J Control Release (2013) 171 (3):349-57. doi: 10.1016/j.jconrel.2013.04.018

12. Resnier P, LeQuinio P, Lautram N, André E, Gaillard C, Bastiat G, et al. Efficient In Vitro Gene Therapy With PEG siRNA Lipid Nanocapsules for Passive Targeting Strategy in Melanoma. Biotechnol J (2014) 9(11):1389-401. doi: 10.1002/biot.201400162

13. Bruniaux J, Gidrol X, Navarro YGF, Sulpice E, Texier-Nogues I. Formulation for the Delivery of Nucleotide Sequences That Can Modulate Endogenous Interfering Rna Mechanisms. Commissariat Energie Atomique (2014). Available at: https://patents.google.com/patent/WO2014032953A1.

14. Kedmi R, Ben-Arie N, Peer D. The Systemic Toxicity of Positively Charged Lipid Nanoparticles and the Role of Toll-Like Receptor 4 in Immune Activation. Biomaterials (2010) 31(26):6867-75. doi: 10.1016/j.biomaterials.2010.05.027

15. Stunault MI, Bories G, Guinamard RR, Ivanov S. Metabolism Plays a Key Role During Macrophage Activation. Mediators Inflamm (2018) 2018:2426138. doi: $10.1155 / 2018 / 2426138$

16. Wculek SK, Khouili SC, Priego E, Heras-Murillo I, Sancho D. Metabolic Control of Dendritic Cell Functions: Digesting Information. Front Immunol (2019) 10:775. doi: 10.3389/fimmu.2019.00775

17. Vangasseri DP, Cui Z, Chen W, Hokey DA, Falo LDJr., Huang L. Immunostimulation of Dendritic Cells by Cationic Liposomes. Mol Membr Biol (2006) 23(5):385-95. doi: 10.1080/09687860600790537

\section{SUPPLEMENTARY MATERIAL}

The Supplementary Material for this article can be found online at: https://www.frontiersin.org/articles/10.3389/fimmu.2021. 722411/full\#supplementary-material

18. de Groot AM, Thanki K, Gangloff M, Falkenberg E, Zeng X, van Bijnen DCJ, et al. Immunogenicity Testing of Lipidoids In Vitro and In Silico: Modulating Lipidoid-Mediated TLR4 Activation by Nanoparticle Design. Mol Ther Nucleic Acids (2018) 11:159-69. doi: 10.1016/j.omtn.2018.02.003

19. Nel AE, Mädler L, Velegol D, Xia T, Hoek EMV, Somasundaran P, et al. Understanding Biophysicochemical Interactions at the Nano-Bio Interface. Nat Materials (2009) 8(7):543-57. doi: 10.1038/nmat2442

20. Blanco E, Shen H, Ferrari M. Principles of Nanoparticle Design for Overcoming Biological Barriers to Drug Delivery. Nat Biotechnol (2015) 33 (9):941-51. doi: 10.1038/nbt.3330

21. Faure M, Villiers CL, Marche PN. Normal Differentiation and Functions of Mouse Dendritic Cells Derived From RAG-Deficient Bone Marrow Progenitors. Cell Immunol (2004) 228(1):8-14. doi: 10.1016/j.cellimm.2004.04.002

22. Chen J, Ellert-Miklaszewska A, Garofalo S, Dey AK, Tang J, Jiang Y, et al. Synthesis and Use of an Amphiphilic Dendrimer for siRNA Delivery Into Primary Immune Cells. Nat Protoc (2021) 16(1):327-51. doi: 10.1038/s41596020-00418-9

23. Courant T, Bayon E, Reynaud-Dougier HL, Villiers C, Menneteau M, Marche PN, et al. Tailoring Nanostructured Lipid Carriers for the Delivery of Protein Antigens: Physicochemical Properties Versus Immunogenicity Studies. Biomaterials (2017) 136:29-42. doi: 10.1016/j.biomaterials.2017.05.001

24. Dey AK, Gonon A, Pécheur E-I, Pezet M, Villiers C, Marche PN. Impact of Gold Nanoparticles on the Functions of Macrophages and Dendritic Cells. Cells (2021) 10(1):96. doi: 10.3390/cells10010096

25. Luo Y, Cook E, Fries BC, Casadevall A. Phagocytic Efficacy of MacrophageLike Cells as a Function of Cell Cycle and Fcgamma Receptors (FcgammaR) and Complement Receptor (CR)3 Expression. Clin Exp Immunol (2006) 145 (2):380-7. doi: 10.1111/j.1365-2249.2006.03132.x

26. Li H, Zhang GX, Chen Y, Xu H, Fitzgerald DC, Zhao Z, et al. CD11c+CD11b+ Dendritic Cells Play an Important Role in Intravenous Tolerance and the Suppression of Experimental Autoimmune Encephalomyelitis. J Immunol (2008) 181(4):2483-93. doi: 10.4049/jimmunol.181.4.2483

27. Zhang X, Goncalves R, Mosser DM. The Isolation and Characterization of Murine Macrophages. Curr Protoc Immunol (2008) 14:14.1. doi: 10.1002\% 2F0471142735.im1401s83

28. Navarro FP, Mittler F, Berger M, Josserand V, Gravier J, Vinet F, et al. Cell Tolerability and Biodistribution in Mice of Indocyanine Green-Loaded Lipid Nanoparticles. J BioMed Nanotechnol (2012) 8(4):594-604. doi: 10.1166/ jbn.2012.1422

29. Hibbitts A, Lucía A, Serrano-Sevilla I, De Matteis L, McArthur M, de la Fuente JM, et al. Co-Delivery of Free Vancomycin and Transcription Factor DecoyNanostructured Lipid Carriers can Enhance Inhibition of Methicillin Resistant Staphylococcus Aureus (MRSA). PloS One (2019) 14(9):e0220684. doi: 10.1371/journal.pone.0220684

30. Tezgel Ö., Szarpak-Jankowska A, Arnould A, Auzély-Velty R, Texier I. Chitosan-Lipid Nanoparticles (CS-LNPs): Application to siRNA Delivery. J Colloid Interface Sci (2018) 510:45-56. doi: 10.1016/j.jcis.2017.09.045

31. Zhang C, Maruggi G, Shan H, Li J. Advances in mRNA Vaccines for Infectious Diseases. Front Immunol (2019) 10:594. doi: 10.3389/fimmu.2019.00594

32. Hinger D, Navarro F, Käch A, Thomann JS, Mittler F, Couffin AC, et al. Photoinduced Effects of M-Tetrahydroxyphenylchlorin Loaded Lipid Nanoemulsions on Multicellular Tumor Spheroids. J Nanobiotechnol (2016) 14(1):68. doi: 10.1186/s12951-016-0221-x

33. Bayon E, Morlieras J, Dereuddre-Bosquet N, Gonon A, Gosse L, Courant T, et al. Overcoming Immunogenicity Issues of HIV P24 Antigen by the Use of Innovative Nanostructured Lipid Carriers as Delivery Systems: Evidences in Mice and non-Human Primates. NPJ Vaccines (2018) 3(1):46. doi: 10.1038/ s41541-018-0086-0 
34. Elouahabi A, Ruysschaert J-M. Formation and Intracellular Trafficking of Lipoplexes and Polyplexes. Mol Ther (2005) 11(3):336-47. doi: 10.1016/j.ymthe.2004.12.006

35. Caracciolo G, Pozzi D, Capriotti AL, Cavaliere C, Piovesana S, Amenitsch H, et al. Lipid Composition: A "Key Factor" for the Rational Manipulation of the Liposome-Protein Corona by Liposome Design. RSC Adv (2015) 5(8):596775. doi: 10.1039/C4RA13335H

36. Moore TL, Rodriguez-Lorenzo L, Hirsch V, Balog S, Urban D, Jud C, et al. Nanoparticle Colloidal Stability in Cell Culture Media and Impact on Cellular Interactions. Chem Soc Rev (2015) 44(17):6287-305. doi: 10.1039/C4CS00487F

37. Caracciolo G, Pozzi D, Capriotti AL, Cavaliere C, Laganà A. Effect of DOPE and Cholesterol on the Protein Adsorption Onto Lipid Nanoparticles. J Nanoparticle Res (2013) 15(3):1498. doi: 10.1007/s11051-013-1498-4

38. Vonarbourg A, Passirani C, Saulnier P, Benoit JP. Parameters Influencing the Stealthiness of Colloidal Drug Delivery Systems. Biomaterials (2006) 27 (24):4356-73. doi: 10.1016/j.biomaterials.2006.03.039

39. Satulovsky J, Carignano MA, Szleifer I. Kinetic and Thermodynamic Control of Protein Adsorption. Proc Natl Acad Sci (2000) 97(16):9037. doi: 10.1073/ pnas. 150236197

40. Szleifer I. Protein Adsorption on Surfaces With Grafted Polymers: A Theoretical Approach. Biophys J (1997) 72(2 Pt 1):595-612. doi: 10.1016/ S0006-3495(97)78698-3

41. Wheeler JJ, Palmer L, Ossanlou M, MacLachlan I, Graham RW, Zhang YP, et al. Stabilized Plasmid-Lipid Particles: Construction and Characterization. Gene Ther (1999) 6(2):271-81. doi: 10.1038/sj.gt.3300821

42. ATCC. Cell Line Misidentification: The Beginning of the End. Nat Rev Cancer (2010) 10(6):441-8. doi: 10.1038/nrc2852

43. Lorsch JR, Collins FS, Lippincott-Schwartz J. Cell Biology. Fixing Problems With Cell Lines. Sci (New York N.Y.) (2014) 346(6216):1452-3. doi: 10.1126/ science. 1259110

44. Deshmane SL, Kremlev S, Amini S, Sawaya BE. Monocyte Chemoattractant Protein-1 (MCP-1): An Overview. J Interferon Cytokine research: Off J Int Soc Interferon Cytokine Res (2009) 29(6):313-26. doi: 10.1089/jir.2008.0027

45. Glista-Baker EE, Taylor AJ, Sayers BC, Thompson EA, Bonner JC. Nickel Nanoparticles Enhance Platelet-Derived Growth Factor-Induced Chemokine Expression by Mesothelial Cells via Prolonged Mitogen-Activated Protein Kinase Activation. Am J Respir Cell Mol Biol (2012) 47(4):552-61. doi: $10.1165 / \mathrm{rcmb} .2012-00230 \mathrm{C}$

46. Rantapää-Dahlqvist S, Boman K, Tarkowski A, Hallmans G. Up Regulation of Monocyte Chemoattractant Protein-1 Expression in Anti-Citrulline Antibody and Immunoglobulin M Rheumatoid Factor Positive Subjects Precedes Onset of Inflammatory Response and Development of Overt Rheumatoid Arthritis. Ann Rheumatic Dis (2007) 66(1):121. doi: 10.1136/ard.2006.057331

47. Ip WK, Wong CK, Lam CWK. Interleukin (IL)-4 and IL-13 Up-Regulate Monocyte Chemoattractant Protein-1 Expression in Human Bronchial Epithelial Cells: Involvement of P38 Mitogen-Activated Protein Kinase, Extracellular Signal-Regulated Kinase 1/2 and Janus Kinase-2 But Not CJun NH2-Terminal Kinase 1/2 Signalling Pathways. Clin Exp Immunol (2006) 145(1):162-72. doi: 10.1111/j.1365-2249.2006.03085.x

48. Van den Bossche J, Baardman J, de Winther MP. Metabolic Characterization of Polarized M1 and M2 Bone Marrow-Derived Macrophages Using Real-Time Extracellular Flux Analysis. J Vis Exp (2015) (105):53424. doi: 10.3791/53424

49. Kelly B, O’Neill LAJ. Metabolic Reprogramming in Macrophages and Dendritic Cells in Innate Immunity. Cell Res (2015) 25(7):771-84. doi: $10.1038 /$ cr.2015.68

50. O’Neill LA, Pearce EJ. Immunometabolism Governs Dendritic Cell and Macrophage Function. J Exp Med (2016) 213(1):15-23. doi: 10.1084/ jem.20151570
51. Fröhlich E. The Role of Surface Charge in Cellular Uptake and Cytotoxicity of Medical Nanoparticles. Int J Nanomed (2012) 7:5577-91. doi: 10.2147/IJN.S36111

52. Henriksen-Lacey M, Christensen D, Bramwell VW, Lindenstrøm T, Agger EM, Andersen P, et al. Comparison of the Depot Effect and Immunogenicity

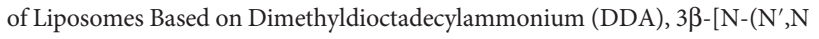
'-Dimethylaminoethane)carbomyl] Cholesterol (DC-Chol), and 1,2-Dioleoyl3-Trimethylammonium Propane (DOTAP): Prolonged Liposome Retention Mediates Stronger Th1 Responses. Mol Pharmaceutics (2011) 8(1):153-61. doi: $10.1021 / \mathrm{mp} 100208 \mathrm{f}$

53. Erni C, Suard C, Freitas S, Dreher D, Merkle HP, Walter E. Evaluation of Cationic Solid Lipid Microparticles as Synthetic Carriers for the Targeted Delivery of Macromolecules to Phagocytic Antigen-Presenting Cells. Biomaterials (2002) 23(23):4667-76. doi: 10.1016/S0142-9612(02)00216-8

54. Fromen CA, Robbins GR, Shen TW, Kai MP, Ting JPY, DeSimone JM. Controlled Analysis of Nanoparticle Charge on Mucosal and Systemic Antibody Responses Following Pulmonary Immunization. Proc Natl Acad Sci (2015) 112(2):488. doi: 10.1073/pnas.1422923112

55. Barillet S, Fattal E, Mura S, Tsapis N, Pallardy M, Hillaireau H, et al. Immunotoxicity of Poly (Lactic-Co-Glycolic Acid) Nanoparticles: Influence of Surface Properties on Dendritic Cell Activation. Nanotoxicology (2019) 13 (5):606-22. doi: 10.1080/17435390.2018.1564078

56. Vasievich EA, Chen W, Huang L. Enantiospecific Adjuvant Activity of Cationic Lipid DOTAP in Cancer Vaccine. Cancer Immunol Immunother (2011) 60(5):629-38. doi: 10.1007/s00262-011-0970-1

57. Erasmus JH, Khandhar AP, Guderian J, Granger B, Archer J, Archer M, et al. A Nanostructured Lipid Carrier for Delivery of a Replicating Viral RNA Provides Single, Low-Dose Protection Against Zika. Mol Ther (2018) 26 (10):2507-22. doi: 10.1016/j.ymthe.2018.07.010

58. Sun M, Li J, Zhang C, Xie Y, Qiao H, Su Z, et al. Arginine-Modified Nanostructured Lipid Carriers With Charge-Reversal and pH-Sensitive Membranolytic Properties for Anticancer Drug Delivery. Adv Healthc Mater (2017) 6(8). doi: 10.1002/adhm.201600693

59. Chen X, Liu L, Jiang C. Charge-Reversal Nanoparticles: Novel Targeted Drug Delivery Carriers. Acta Pharm Sinica B (2016) 6(4):261-7. doi: 10.1016/ j.apsb.2016.05.011

60. Han L, Zhao J, Zhang X, Cao W, Hu X, Zou G, et al. Enhanced siRNA Delivery and Silencing Gold-Chitosan Nanosystem With Surface Charge-Reversal Polymer Assembly and Good Biocompatibility. ACS Nano (2012) 6 (8):7340-51. doi: $10.1021 / \mathrm{nn} 3024688$

Conflict of Interest: The authors declare that the research was conducted in the absence of any commercial or financial relationships that could be construed as a potential conflict of interest.

Publisher's Note: All claims expressed in this article are solely those of the authors and do not necessarily represent those of their affiliated organizations, or those of the publisher, the editors and the reviewers. Any product that may be evaluated in this article, or claim that may be made by its manufacturer, is not guaranteed or endorsed by the publisher.

Copyright (®) 2021 Dey, Nougarède, Clément, Fournier, Jouvin-Marche, Escudé, Jary, Navarro and Marche. This is an open-access article distributed under the terms of the Creative Commons Attribution License (CC BY). The use, distribution or reproduction in other forums is permitted, provided the original author(s) and the copyright owner(s) are credited and that the original publication in this journal is cited, in accordance with accepted academic practice. No use, distribution or reproduction is permitted which does not comply with these terms. 